\title{
The use of direct inverse maps to solve material identification problems: pitfalls
} and solutions

\author{
Erfan Asaadi ${ }^{1}$, Daniel N. Wilke ${ }^{2}$, P. Stephan Heyns ${ }^{3}$, Schalk Kok ${ }^{4}$ \\ 1-4 Centre for Asset Integrity Management, Department of Mechanical and Aeronautical \\ Engineering, University of Pretoria, Pretoria, South Africa \\ ${ }^{1}$ Corresponding Author: Tel:+27124202432. E-mail address: er.asaadi@gmail.com \\ ${ }^{2}$ nico.wilke@up.ac.za \\ ${ }^{3}$ stephan.heyns@up.ac.za \\ ${ }^{4}$ schalk.kok@up.ac.z
}

\begin{abstract}
Material parameter identification is a technique that is used to calibrate material models, often a precursor to perform an industrial analysis. Conventional material parameter identification methods estimate the material parameters for a material model by solving an optimisation problem. An alternative but lesser-known approach, called a direct inverse map, directly maps the measured response to the parameters of a material model. In this study we investigate the potential pitfalls of the well-known stochastic noise and lesser-known model errors when constructing direct inverse maps. We show how to address these problems, explaining in particular the importance of projecting the measured response onto the domain of the simulated responses before mapping it to the material parameters. This paper concludes by proposing partial least squares regression as an elegant and computationally efficient approach to address stochastic and systematic (model) errors. This paper also gives insight into the nature of the inverse problem under consideration.
\end{abstract}

Keywords: Inverse problem; Inverse mapping; Material parameter identification; Partial least squares regression; Principal component analysis; Radial basis function approximation

\section{Introduction}

In the context of this study, the definition of a system, which produces a response based on some input parameters, is restricted to being either a finite element analysis (FEA) or an actual experiment. Often the system parameters are difficult or impossible to measure directly, although the system response can be measured readily. An inverse identification problem, also called an inverse problem, is generally defined as an approach to identify or infer the system parameters from the measured system response (Stavroulakis et al. 2003). The system parameters obtained from an inverse analysis are often necessary for a simulation of a more complicated system. For this reason, dependable inverse identification methods also play a vital role in conducting reliable FEA. The systematic adoption of inverse identification methods may reduce the tendency to add complexity to 
finite element models (FEMs) whenever the model's output does not match the measured response of the system, whereas the original model might have been adequate if it had been calibrated properly.

In order to infer the material parameters (the system parameters) from the measured response (the system response), it is common practice to first simulate the measured response using FEA. The simulated measured responses (the simulation responses) are then used to calibrate the material model using inverse analysis.

The measured response of the system $\mathbf{Y}_{\mathbf{0}}{ }^{\mathrm{i}}$ (superscript $\mathrm{i}$ refers to the $\mathrm{i}^{\text {th }}$ repetition of the measurement and without superscript generally means the response of the system) can be assumed to be a combination of the true system response $\widetilde{\mathbf{Y}}$, combined with some measurement errors $\mathbf{e}_{\boldsymbol{s} \boldsymbol{e}}^{\boldsymbol{i}}$ (aleatoric uncertainty) which we call stochastic noise. This is expressed as

$$
\mathbf{Y}_{0}^{i}=\widetilde{\mathbf{Y}}+\mathbf{e}_{s e}^{i} .
$$

Note that the true system response $\widetilde{\mathbf{Y}}$ is assumed to exist, but it is unknown. Should we repeat a measurement a number of times, we could hypothetically generate a distribution of measurement errors (stochastic noise), which we assume have zero mean. Such a set of possible measured responses $\mathbf{Y}_{\mathbf{0}}$ defines the space $\mathbb{R}_{\text {exp }}^{L}$ of measured responses.

The simulated response $\mathcal{R}(\boldsymbol{\theta})=\mathbf{Y}$ depends on the simulation parameters $\boldsymbol{\theta}$. Simulating the responses choosing different parameters $\boldsymbol{\theta}^{i}$ defines the space of simulated responses $\mathbb{R}_{\text {sim }}^{L}$. Even when we consider the simulation parameters $\boldsymbol{\theta}^{*}$ that best predict the true system response, there remains a difference between the simulated and measured responses. This difference is referred to as the model error $\mathbf{e}_{\mathbf{m e}}$, (epistemic uncertainty), as defined in

$$
\mathcal{R}\left(\boldsymbol{\theta}^{*}\right)+\mathbf{e}_{\mathbf{m e}}=\widetilde{\mathbf{Y}} .
$$

The lower the model error the better the simulated response is able to approximate the measured experimental response (Oden and Prudhomme 2002). Hence, the simulated response $\mathcal{R}(\boldsymbol{\theta})$ has a limited ability to capture the true measured response. Fig. 1 depicts the measured response space, the simulated space as well as the measured response space projected onto the simulated space. The difference between the projected response space and the simulated response space indicates the limited ability of the simulated response space to represent the measured response space.

From equation (1) and equation (2) the relationship between the measured response and the simulated response is given by

$$
\mathbf{Y}_{\mathbf{0}}=\mathcal{R}\left(\boldsymbol{\theta}^{*}\right)+\mathbf{e}_{\mathbf{m e}}+\mathbf{e}_{\mathbf{s e}} .
$$

The aim of inverse analysis is to estimate the material model parameters $\boldsymbol{\theta}^{*}$ that when used in the simulation $\mathcal{R}\left(\boldsymbol{\theta}^{*}\right)$, best predict the measured response $\mathbf{Y}_{\mathbf{0}}$. Due to the nonlinear nature of the problem this usually is done iteratively by minimising the sum of the squared errors $e$ between the two responses, given by

$$
e=\left(\mathbf{Y}_{\mathbf{0}}-\mathcal{R}\left(\boldsymbol{\theta}^{*}\right)\right)^{T}\left(\mathbf{Y}_{\mathbf{0}}-\mathcal{R}\left(\boldsymbol{\theta}^{*}\right)\right) .
$$

Notice that $e$ includes both the stochastic noise and the model error. We refer to this approach as the (conventional) forward inverse method which is common practice for identifying material parameters (Aguir et al. 2011 ; Aguir et al. 2008 ; Asaadi and Heyns 2016 ; Bolzon and Talassi 2013 ; Brigham and Aquino 2007 ; Degroote et al. 2012 ; Harb et al. 2014 ; Munoz-Sánchez et al. 
The errors feature in the forward inverse method when computing the sum of squared errors between the simulated response (which contains the model error) and the measured response (which contains the stochastic noise). The forward inverse method finds the best fit of the simulated response to the measured response, even if the simulation model does not capture the physics exactly (Aguir et al. 2011 ; Zribi et al. 2013). In other words, optimisation gives the optimal projection of the measured response onto the simulated response space, while the difference between the two responses is indicative of the information present in the measured response that could not be captured by the simulation.

However, the computational cost associated with conducting an FEA for each function evaluation (a sum of squared errors computation) and multiple FEAs for a finite difference gradient vector approximation might be prohibitively expensive. Alternatively, response surfaces or surrogates can be used to approximate only the sum of squared errors. Another option is to use multiple surrogates to estimate the simulation responses directly. The sum of squared errors can then be computed from the surrogate responses. Popular strategies include artificial neural networks (Aguir et al. 2011 ; Aguir et al. 2008 ; Asaadi and Heyns 2016 ; Brigham and Aquino 2007 ; Hambli and Guerin 2003 ; Zhang et al. 2013) or radial basis functions (RBF) (Bolzon and Talassi 2013 ; Munoz-Sánchez et al. 2011). Fig. 2(a) portrays an outline of forward inverse parameter identification, using surrogates. However, neither the construction of an accurate surrogate model, nor the minimisation of the estimated error is a straightforward task.

Regarding the construction of a surrogate, a set of simulated responses for chosen input parameters are constructed and split into a training set and a validation set. The training set contains the information blended by a surrogate to estimate unseen responses from new input parameters. Surrogates have unknown parameters which influence the accuracy of the estimated response. The validation set is used to compute the estimated response error in the validation set. This error is then minimised to obtain the optimal parameters for the surrogate.

It would be beneficial if the complexity of the forward surrogate-based inverse problem could be partly reduced. A lesser-known alternative to solve the material parameter identification problem is the backward inverse identification method, or the direct inverse map (Abbassi et al. 2013 ; Chamekh 2009 ; Huber and Tsakmakis 2001). The true direct inverse map $\tilde{\mathcal{R}}^{-1}(\mathbf{Y})$ is constructed to directly map the measured response $\mathbf{Y}_{\mathbf{0}}$ to the system parameters $\boldsymbol{\theta}^{*}$. In general the true direct inverse map is defined by

$$
\tilde{\mathcal{R}}^{-1}\left(\mathbf{Y}_{\mathbf{0}}\right)=\boldsymbol{\theta}^{*}
$$

and does not require any form of iteration or optimization. This is in stark contrast to the forward inverse method that estimates the measured response from the material model parameters by iteratively reducing the sum of squared errors between the simulated and measured response.

In order to construct the direct inverse map, we require data. If we make use of simulated data, we are constructing a direct inverse map of the FEM, $\mathcal{R}^{-1}$. This process is outlined schematically in Fig. 2(b). However, there are several aspects to consider which are not well known, and their importance is not well documented in open literature.

Note that $\mathbf{Y}_{\mathbf{0}}$ includes the stochastic noise and the model error. Therefore when $\mathbf{Y}_{\mathbf{0}}$ is mapped to the corresponding material model parameters using the direct inverse map $\mathcal{R}^{-1}$, the errors are also mapped as given by 


$$
\left.\mathcal{R}^{-1}\left(\mathbf{Y}_{\mathbf{0}}\right)=\mathcal{R}^{-1}\left(\mathcal{R}\left(\boldsymbol{\theta}^{*}\right)+\mathbf{e}_{\mathbf{m e}}+\mathbf{e}_{\mathbf{s e}}\right)\right) .
$$

However, the simulated responses used to construct $\mathcal{R}^{-1}$ are free from stochastic noise and model errors i.e. the direct inverse map was constructed to only map $\mathbf{Y}=\mathcal{R}(\boldsymbol{\theta})$. Therefore, the differences between the measured response space $\mathbb{R}_{\text {exp }}^{L}$ and the simulated response space $\mathbb{R}_{\text {sim }}^{L}$ become critical to consider. Fig. 1 depicts the measured response space $\mathbb{R}_{\text {exp }}^{L}$ and the simulated response space $\mathbb{R}_{\text {sim. }}^{L}$, from which it is clear that not all of the measured responses can be simulated exactly. This in general includes the true experimental response $\widetilde{\mathbf{Y}}$. Mapping these errors may result in nonsensical parameter estimates (which we refer to as a lack of robustness), to be demonstrated in our numerical investigation. In this study we demonstrate that it is essential to be robust in the presence of i) stochastic noise and ii) model errors. This is critical to avoid nonsensical estimates. Robustness against stochastic noise is fairly well understood and elegantly addressed by regularisation in the form of regression, but robustness against model errors is not well understood in material parameter identification, e.g. see (Abbassi et al. 2013 ; Chamekh 2009; Huber and Tsakmakis 2001).

To be robust against the model error, the direct inverse map requires a projection step. The key idea is to construct the direct inverse map such that only the information of $\mathbb{R}_{\text {exp }}^{L}$ present in $\mathbb{R}_{\text {sim }}^{L}$ is mapped, which is indicated by the grey area in Fig. 1. The measured response $\mathbf{Y}_{\mathbf{0}}$ is projected to the simulated space, which gives the projected measured response $\mathbf{Y}_{\mathbf{0}}^{\mathbf{p}}$. We then map $\mathbf{Y}_{\mathbf{0}}^{\mathbf{p}}$, as opposed to $\mathbf{Y}_{\mathbf{0}}$ using the direct inverse map $\mathcal{R}^{-1}$. In this study we project the measured response space onto the simulated response space using reduced basis strategies.

The focus of this study is to demonstrate the importance of regression and projection, in the construction of the direct inverse map to enhance its accuracy and robustness. We provide theoretical background for direct inverse map construction in Section 2. The model examples are described in Section 3, in which we demonstrate the importance of considering stochastic noise as well as model error in the construction of direct inverse maps to ensure accurate mappings from the measured response to the system parameters. It is demonstrated that regression and projection enhance the robustness of the inverse mapping in the presence of stochastic noise and model error. It is shown that partial least squares regression (PLSR) is a convenient starting point for constructing direct inverse maps because it robustly handles both the stochastic noise and model errors, and it is computationally efficient. Section 4 concludes the study.

\section{Direct inverse map}

\subsection{Nonlinear surrogates}

In order to construct a surrogate model, numerous blending strategies exist e.g. polynomial, ANN and RBF. Although ANN is a popular choice for direct inverse maps, we opt for the radial basis function approximation due to its flexibility, simplicity and the intuitive understanding of its parameters. For completeness, we give a brief outline of the RBF approximation.

The RBF is one of the most effective methods for approximating multivariate functions from sparse data by computing linear combinations of non-linear basis functions (Buhmann 2004). It is defined as 


$$
\theta(\mathbf{Y})=\sum_{n=1}^{k} w_{n} \varphi\left(\left\|\mathbf{Y}-\mathbf{Y}_{\mathrm{n}}\right\|^{2}\right)
$$

where $\theta(\mathbf{Y})$ is the scalar approximation of the function/data, $w_{n}$ is the weight, $\varphi$ is the basis function, $\|\ldots\|$ is the Euclidean norm, $\mathbf{Y}$ is the input vector and vector $\mathbf{Y}_{\mathrm{n}}$ contains the centres for the $k$ basis functions. One of the most popular basis functions is the Gaussian function, defined by

$$
\varphi=\exp \left(-\gamma r^{2}\right) \quad: \quad r=\left\|\mathbf{Y}-\mathbf{Y}_{n}\right\|
$$

where $\gamma$, which is called the shape function parameter or range parameter, defines the spread of the basis function. A large $\gamma$ indicates a localised influence in the $N$-dimensional response space and a small $\gamma$ indicates a global influence in the $\mathrm{N}$-dimensional response space. It is vital to select the proper $\gamma$ to ensure the accuracy of the approximated model on the validation set. In this study $\gamma$ is selected using particle swarm optimisation (Chen 2009) with 7 restarts to find the optimum $\gamma$ so that the RBF minimises the simulation response error for a random set of 20 validation points. This choice results in a computationally cheap and unconstrained univariate minimisation problem where only one $\gamma$ per RBF is considered. The RBF surrogate model using $\mathrm{k}$ centres and the Gaussian function is given by

$$
\theta(\mathbf{Y})=\sum_{n=1}^{k} w_{n} \exp \left(-\gamma\left\|\mathbf{Y}-\mathbf{Y}_{\mathrm{n}}\right\|^{2}\right)
$$

To find the weights $w_{n}$, the inputs $\mathbf{Y}_{\mathrm{i}}$ and the outputs $\theta_{i}$ of the $L$ training points are substituted into the RBF approximation (10) to give a system of $L$ equations and k unknowns,

$$
\underbrace{\left[\begin{array}{lll}
\exp \left(-\gamma\left\|\mathbf{Y}_{1}-\mathbf{Y}_{1}\right\|^{2}\right) & \ldots & \exp \left(-\gamma\left\|\mathbf{Y}_{1}-\mathbf{Y}_{\mathrm{k}}\right\|^{2}\right) \\
\exp \left(-\gamma\left\|\mathbf{Y}_{2}-\mathbf{Y}_{1}\right\|^{2}\right) & \ldots & \exp \left(-\gamma\left\|\mathbf{Y}_{2}-\mathbf{Y}_{\mathrm{k}}\right\|^{2}\right) \\
\exp \left(-\gamma\left\|\mathbf{Y}_{\mathrm{L}}-\mathbf{Y}_{1}\right\|^{2}\right) & \ldots & \exp \left(-\gamma\left\|\mathbf{Y}_{\mathrm{L}}-\mathbf{Y}_{\mathrm{k}}\right\|^{2}\right.
\end{array}\right]}_{\varnothing} \underbrace{\left[\begin{array}{c}
w_{1} \\
w_{2} \\
\ldots \\
w_{k}
\end{array}\right]}_{\mathbf{W}}=\underbrace{\left[\begin{array}{c}
\theta_{1} \\
\theta_{2} \\
\ldots \\
\theta_{L}
\end{array}\right]}_{\boldsymbol{\theta}} .
$$

When $\mathrm{k}$ is equal to $\mathrm{L}$, we construct an $\mathrm{RBF}$ interpolation function, which allows us to compute $\mathbf{W}$ by solving the linear system

$$
\varnothing \mathbf{W}=\boldsymbol{\theta} .
$$

For $k$ less than $L$ we have RBF least squares regression, from which $\mathbf{W}$ can be computed by solving the linear system:

$$
\emptyset^{\mathbf{T}} \emptyset \mathbf{W}=\emptyset^{\mathbf{T}} \boldsymbol{\theta}
$$

Surrogate models can also be constructed based on either the original data vector $\mathbf{Y}$, or based on projected data (Asaadi and Heyns 2016 ; Gogu and Passieux 2013). Therefore, some projection methods are explained next.

\subsection{Principal component analysis (PCA)}

Principal component analysis (PCA) allows a complex multivariate dataset $\mathrm{Y}$ to be expressed in a transformed co-ordinate system in such a way that the dataset projected onto the first transformed 
co-ordinate (principal component) describes the most variance in the data, the second co-ordinate the second-most variance, and progressively less as the number of co-ordinates increases (Jolliffe 2002). This feature makes PCA ideal for data reduction by considering fewer principal components than the dimensionality of the data set, knowing that the information contained in the chosen principal components describes a large fraction of the total information in the dataset. This allows the data to be expressed as a linear combination of the principal directions. The principal directions are the directions of the principal components in the original co-ordinate system. The $L \times N$ dataset Ý with $L$ observations each having dimension $N$ can be expressed by

$$
\dot{\mathbf{Y}} \approx \mathbf{T}_{\mathrm{A}} \mathbf{P}_{\mathrm{A}}^{\mathrm{T}}
$$

where $\mathbf{T}_{\mathrm{A}}(L \times A)$ and $\mathbf{P}_{\mathrm{A}}(N \times A$, with $A \leq N)$ represent the principal component matrix and the principal direction matrix respectively. Each column of $\mathbf{T}_{\mathrm{A}}$ and $\mathbf{P}_{\mathrm{A}}$ is related to a principal component in the transformed co-ordinate system, while the rows are related to the original coordinate system. Should the number of columns (principal directions) be less than the rows $(N)$, we only approximate the dataset $Y$. This is usually the case. PCA has become a standard tool in most linear algebra packages and numerical libraries, e.g. Matlab , $\mathrm{R}$ and Python SciPy. We compute the principal directions and components by conducting a spectral decomposition of $\hat{Y}^{\mathrm{T}} \hat{\mathrm{Y}}$. Additional information is available from (Andrade-Garda 2009a ; Jolliffe 2002 ; Sun 2008).

\subsection{Linear surrogates}

Since the principal directions represent a large fraction of the non-linearity present in the dataset Ý vector, it is worth considering whether a non-linear mapping between principal components is essential. The linear mapping from the principal component space of the dataset $Y$ to the parameter space is known as principal component regression (PCR) (Andrade-Garda 2009a).

PCR finds the principal directions and components that describe the most variation in the dataset Ý, in isolation from the parameters $\theta$. An alternative to PCR is partial least-squares regression (PLSR), a technique that finds partial least-square directions (loadings) in the system response $Y$ that describes the most variation in the system parameter data $\boldsymbol{\theta}$ (Andrade-Garda 2009b ; Sun 2008). Hence, the system response and system parameters are not treated in isolation. This technique aims from the outset to map from the system response to the system parameters.

When considering the matrix of system responses Ý and the matrix of system parameters $\boldsymbol{\theta}$, partial least-squares regression computes the partial least square directions (loadings) and partial least square components (scores) by conducting a singular value decomposition of $\hat{Y}^{\mathrm{T}} \boldsymbol{\theta}$. The singular values are decomposed into the scores of the system responses $\mathbf{T}$ and system parameters $\mathbf{U}$. Similarly, the singular directions are decomposed into loadings of the system responses $\mathbf{P}$ and system parameters Q (Kaspar and Ray 1993). The original system responses and parameters can then be expressed as

$$
\hat{\mathbf{Y}}=\mathbf{T} \mathbf{P}^{\mathrm{T}}, \quad \boldsymbol{\theta}=\mathbf{U} \mathbf{Q}^{\mathrm{T}}
$$

The potential benefit of PLSR is self-evident but researchers specializing in material parameter identification still have to adopt it. 


\section{Numerical examples}

In this Section we solve two material parameter identification problems, using direct inverse map. In the first problem a tube bulge test is used to identify the stress-strain curve of tubular materials. The second example identifies Voce material model parameters which describe experimental data for commercially pure Titanium.

\subsection{Tube bulge test for material parameter identification}

The model problem for this study is to identify the stress-strain curve of tubular materials from a tube bulge test. The tube bulge test is a popular experiment in which internal pressure is applied to a partially constrained tubular sample (Asaadi and Heyns 2016 ; Bortot et al. 2008 ; Lianfa and Cheng 2008 ; Xu et al. 2008 ; Zribi et al. 2013). We measure the hoop strain on the bulge pole as a function of internal pressure. The aim of the material identification problem is to relate the measured hoop strain-internal pressure response to the stress-strain behaviour of the material.

We conduct our study in a virtual environment where finite element (FE) simulations of the bulge test are conducted for selected material parameters to obtain the hoop-strain internal pressure response. These simulations are used to construct the required data for the training set and the validation set as well as the experimental data. The simulated experimental data (virtual experiment) is stored in a test set. We restrict ourselves in this study to simulated experimental data as opposed to actual experimental data. The benefit of simulating the experimental data is that the type and severity of errors can be controlled. This allows us to gain a systematic and proper understanding of how the errors present in the experimental data influence the quality of the direct inverse maps.

\subsubsection{Finite element simulations of the tube bulge test}

The axisymmetric FEM of the tube bulge test is presented in Fig. 3. The computational cost of the axisymmetric model is further reduced by reflection symmetry across the vertical axis, using the appropriate vertical roller boundary conditions as indicated. The length of the tube is $120 \mathrm{~mm}$, the diameter is $63.5 \mathrm{~mm}$ and the tube is $1.5 \mathrm{~mm}$ thick. Seventy quadrilateral axisymmetric elements along the length and four elements through the thickness are considered. The tube is subjected to an internal pressure that is linearly increased from zero to $24 \mathrm{MPa}$ over the simulation timesteps. Part of the tube is constrained by a die with corner radius $5 \mathrm{~mm}$ to restrict movement in the axial direction only. The contact between the die and the tube is modelled as frictionless. Simulated hoop strain on the peak of the bulged area is recorded at 27 substeps as the simulation response, see Fig. 3. The process is simulated by using the finite element package Ansys Mechanical. In this study, we model the hardening behaviour of the material using the Ludwik equation

$$
\sigma=Y+K \varepsilon^{n},
$$

where $\sigma$ is the true Von Mises stress, $\varepsilon$ is the true equivalent plastic strain and $Y, K$ and $n$ are model parameters. A Young's modulus of $200 \mathrm{GPa}$ and a Poisson ratio of 0.3 is used for the elastic part of all material models. Although numerous material models are available, an appropriate material model is usually selected on the basis of engineering experience. An appropriate material model will closely resemble the actual stress-strain response of the material in the experiment, resulting in a small model error. Larger model errors arise when the set of possible stress-strain responses of a model differ significantly from the stress-strain response of the real material. For the sake of clarity, notice that we use the same structural parameters, such as pipe diameter, wall thickness and 
boundary conditions and operational pressure for all the simulations. If the actual structural and operational parameters differ from the parameters used in the simulation, this will contribute to model error.

\subsubsection{Direct inverse map construction}

Two options are available to construct the direct inverse map for material parameter identification. The direct inverse map can be constructed to map the hoop strain-internal pressure response to the Ludwik model parameters. The stress-strain response can then be computed from the model parameters. Alternatively, one can relate the hoop strain-internal pressure response to the stressstrain curve, from which the model parameters can be inferred. We consider the former approach in this study.

The data for the training and validation sets is computed by choosing a set of simulated materials, each defined by a choice for the Ludwik parameters $(Y, K, n)$. Then a finite element simulation is conducted for each material in the set to obtain the hoop strain-internal pressure response. Once all the finite element simulations have been conducted, numerous hoop strain-internal pressure responses are obtained for various simulated materials, each given by their material model and material parameters. The hoop strain-internal pressure data serves as the input and the material parameters serve as the output in the construction of the direct inverse map.

An initial set of 2000 Ludwik material parameter settings $(Y, K, n)$ is generated using the Latin hypercube sampling available in Matlab. The ranges used for the material parameters are $Y \in[170$; 210] $\mathrm{MPa}, K \in[630 ; 1170] \mathrm{MPa}$ and $n \in[0.35 ; 0.65]$. The material parameters are represented by $M$ dimensional vectors, with $M=3$. We were able to conduct 934 successful simulations, since the FE analyses for some material parameters settings did not converge owing to insufficient workhardening. Since the hoop strain was recorded at 27 internal pressure loads for each simulation, the system response is represented by an $N$ dimensional vector, with $N=27$. The 934 successful simulations are split into training, validation and test sets, where each set contains unique points.

Since the direct inverse map is a surrogate, its construction follows the usual two-step process. Firstly, a blending strategy is chosen and secondly, the optimal blending or range parameters are computed.

We construct a training set with $L$ points, where the simulated responses ( $N \times 1$ vectors) and the material parameters $(M \times 1$ vectors $)$ are both known. An RBF is used to interpolate between these $L$ simulated responses to estimate one of the $M$ material parameters, by directly mapping the simulated response to a material parameter. In total, we construct $M$ RBF functions to estimate the $M$ material parameters.

We have to find the optimal range parameter for each constructed RBF. The range parameter determines how information at different length scales is blended or how information diminishes over some distance (norm) in the $\mathrm{N}$-dimensional simulation response space. We choose to minimise the validation error, similar to the strategy used in (Fasshauer and Zhang 2007). We estimate the material parameters at these $V$ points and find the range parameter that best predicts the material parameters from the simulated responses. Finding the optimal range parameter is crucial when constructing an accurate direct inverse map. 


\subsubsection{Studying the sensitivity of the direct inverse map}

We start with test sets without stochastic noise and without model errors. We systematically introduce noise into the test set to investigate the effect of the various types of noise as well as the severity of the noise. We then investigate the effect of model errors. We also demonstrate some remedial actions that can be taken in the construction of the direct inverse map to enhance the robustness of the map in the presence of stochastic noise and model error.

\section{Effect of stochastic noise in the experimental response}

We start by considering the sensitivity of a naively constructed direct inverse map to the presence of stochastic noise in the measured hoop strain-internal pressure response. Note that we only consider stochastic noise with a zero mean as it is clear that having stochastic noise with a non-zero mean would off-set the result.

We construct three interpolating RBFs, hence the number of centres $k$ equals the number of points $L$ in the training set. We map the hoop strain-internal pressure response to each of the three material parameters of the Ludwik material model. The estimated material parameters are then used in the Ludwik model to construct the estimated stress-strain response of the material. If the actual stressstrain response is known, we can compute the prediction error of the direct inverse map.

For our numerical experiment, we randomly select 100 points from the 934 simulated responses, which we divide as follows: $L=60$ (training points), $V=20$ (validation points) and $T=20$ (test points). Our naively constructed direct inverse map uses the simulated data of $L$ random hoop strain-internal pressure vectors and $V$ validation vectors, without the presence of any noise. Then we use a multistart PSO algorithm to find the optimal range parameter in the RBF that best predicts the response at $V$ random validation points. Finally, we report the prediction error at the $T$ test points. Since experimental data always has measurement errors, our test points should reflect that aspect to ensure that we accurately assess the performance of the direct inverse map. Accordingly, we also consider the $T$ test points with $2 \%$ uniform stochastic noise added to the hoop strain-internal pressure response of the test points. We repeat this process 200 times to quantify statistically the sensitivity of the constructed direct inverse map.

Fig. 4 depicts the probability that the direct inverse map predicts the stress-strain response below a specific percentage error, for test points without noise and test points with $\pm 2 \%$ uniform random noise added. Note that the direct inverse map is able to accurately predict the material parameters from test points without noise. It is clear from Fig. 4, however, that there is a significant degradation in the prediction accuracy when stochastic noise is added to the test points.

This degradation in prediction performance is due to the numerical ill-conditioning of the matrix $\emptyset$ in equation (11), as mentioned by (Uhl 2007). Although not shown, the range parameter for the direct inverse map is optimal for global spatial support of information in the absence of noise in the validation set. This global spatial support increases the prediction accuracy in the absence of noise, but also leads to the numerical ill-conditioning of $\emptyset$. This results in a highly sensitive and therefore an ill-posed direct inverse map. Here the stochastic noise present in the test set is amplified by the numerical ill-conditioning, resulting in worse prediction for noisy data.

In equation (11), an ill-conditioned $\varnothing$ matrix coincides with large $\mathbf{W}$ parameters that are orders of magnitude larger than the actual values that they aim to interpolate. Consequently small variations in $\mathbf{Y}$ result in large variations of $\theta(\mathbf{Y})$ in equation (9), making the direct inverse map ill-posed and therefore highly sensitive. To address this ill-posed issue, we need to ensure that better-conditioned 
$\emptyset$ matrices are computed when solving equation (11), but not to the extent that this compromises the accuracy of the global spatial support of information.

\section{Regularisation}

We consider some strategies that improve the conditioning of $\varnothing$ just enough to also improve the accuracy of the direct inverse map. These strategies are also known as regularisation strategies (Uhl 2007). Note that this is not an exhaustive list of approaches, but merely gives some common approaches to demonstrate that the accuracy of the direct inverse map may improve when the conditioning of $\varnothing$ is improved.

A natural regularisation approach might be to incorporate stochastic noise in the validation points when we aim to find the optimal range parameter; this is popular in ANN strategies (An 1996 ; Brown et al. 2003). Accordingly, we evaluate the prediction accuracy of the direct inverse map with the presence of stochastic noise when solving for the optimal range parameters. We introduce noise by repeating the points in the validation set twice, and adding stochastic noise to the repeated points. Therefore, our validation set contains points without stochastic noise (one-third of the points) as well as points with random stochastic noise (two-thirds of the points). The RBF is however still constructed to interpolate using the noiseless training set. The test set, which has $2 \%$ uniformly distributed stochastic noise added, is still used to compute the prediction accuracy for the response.

The true range and distribution of the stochastic noise present in the experimental response are unknown. For this reason, we consider various scenarios. The first three validation sets have $1 \%$, $2 \%$ and $3 \%$ uniform stochastic noise added to the repeated data points. We also consider normally distributed stochastic noise with a standard deviation of 0.01 in the validation set. The noiseless and noisy data points in the validation set are required to predict the same material parameters when finding the optimal range parameters.

Fig. 5 presents the results of the prediction accuracy of the direct inverse map constructed with or without noise in the validation set. We note that adding noise to the validation set has a major effect on prediction accuracy, while the amount or distribution of the added noise only has a minor effect on prediction accuracy. Also note that matching the exact percentage of stochastic noise in the validation set to the percentage of stochastic noise in the test set seems to be only marginally more accurate. We also observe a slight degradation in prediction accuracy when the stochastic noise is overestimated (3\% stochastic noise case).

Alternatively, the noise can be introduced in the training set during the computation of the W parameters of the RBF function, as opposed to the validation set. So far, we computed $\mathbf{W}$ by interpolation, i.e. the training points and the locations where the response was matched were kept the same, resulting in the system described by equation (11). However, as a simple demonstration of constructing the RBF by regression, using equation (12), we increase the training set by repeating the points in the training set twice and adding stochastic noise to the simulated response of the repeated points, while keeping the material parameters the same. The noiseless and noisy simulated responses are required to predict the same material parameters. We keep the number of radial basis functions and centres of the radial basis functions the same as those used to construct the RBF interpolation, i.e. the same number and position of the noiseless training points. The range parameter is estimated by using one of two validation sets. One set contains only noiseless data points and the other set contains both noiseless data and $2 \%$ uniformly distributed random noise added to the same data. 
Fig. 6 depicts the prediction error of the RBFs constructed by regression using $2 \%$ uniformly distributed stochastic noise in the training set. The range parameter is estimated for both the noisy and the noiseless validation sets. The prediction accuracy is contrasted against the interpolation $\mathrm{RBF}$ with the optimal range parameters computed by using $2 \%$ stochastic noise in the validation set. It is clear that regression is insensitive to whether or not noise is present in the validation set. Regression performs significantly better than the interpolation RBF with $2 \%$ stochastic noise in the validation sets. The associated distribution of the stress-strain responses for a randomly selected test point is depicted in Fig. 7, for 100 instances of adding 2\% uniform random stochastic noise to the hoop strain-pressure data of the test point. It is clear that the ill-posed nature of the interpolation map constructed with the noiseless validation set is significantly higher than the regression map constructed with $2 \%$ stochastic noise in the validation set and training set.

Both methods considered here are simple demonstrations of the benefits of considering stochastic noise when constructing surrogates for the direct inverse map. We emphasise that more elaborate strategies could be used to select the optimal number of basis functions and the centre positions for the regression RBF, e.g. k-mean clustering (Hastie et al. 2003). Alternative approaches to improve the conditioning of $\varnothing$ e.g. singular value decomposition (Oliver et al. 2008) and Tikhonov regularisation (Tikhonov 1977 ; Uhl 2007)) might supplement or improve our elementary strategies. It is not our aim to investigate or present the most effective strategy for this problem but merely to demonstrate the importance and benefits of considering stochastic noise when constructing a direct inverse map. This is particularly relevant because of the inevitable presence of stochastic noise in the measured response.

\section{Mismatch between the simulation model and reality (Model error)}

Stochastic noise is not the only consideration when constructing a direct inverse map, since the selected model used for constructing the training and validation sets might not be able to predict the experimental response accurately. Since models only approximate reality, there is always some mismatch between the response of the model and the experimental response. We demonstrate the degradation of the prediction accuracy when some model mismatch is present between the simulated and measured responses. This degradation is greater than anticipated for an associated model mismatch, and becomes amplified as the model mismatch increases. We demonstrate some strategies to improve the robustness of predictions, to match the expected accuracy for a given model mismatch.

The treatment of the model error is not prominent in the literature, since the model error is not usually of concern in the classical forward surrogate-based inverse analysis. In contrast, the model error has to be explicitly dealt with when constructing direct inverse maps. First we show that if care is not exercised, the system parameters predicted from the experimental response can be severely deficient.

To quantify the effect of the model error, we compute the training and validation points using the Ludwik equation as before. However, the test points, playing the role of virtual experimental data, are generated by using the bi-linear model given by

$$
\begin{array}{cc}
\sigma=\sigma_{1}+T_{1} \varepsilon, & \text { if } \varepsilon \leq \varepsilon_{0} \\
\sigma=\sigma_{2}+T_{2}\left(\varepsilon-\varepsilon_{0}\right), & \text { if } \varepsilon>\varepsilon_{0}
\end{array}
$$

with $\sigma_{1}, \sigma_{2}, T_{1}$ and $T_{2}$ the model parameters. $\varepsilon_{0}$ is computed from the intersection of the two linear sections. Two test points are constructed using the chosen material parameters shown in Tab. 1 Simulations for each bi-linear test point are conducted to obtain the hoop strain-internal pressure 
responses. To quantify how much the model mismatch contributes to the mapped stress-strain difference, we find the two Ludwik stress-strain models with associated parameters that best describe the bilinear stress-strain response for the two test points. We therefore optimally project (fit) the bi-linear stress-strain response onto the Ludwik stress-strain response space by minimising the sum of squares error between the two stress-strain curves. The associated optimally projected Ludwik parameters are presented in Tab. 1. It should be noted that the optimally projected Ludwik parameters are within the parameter bounds used for constructing the training set data. Therefore we (naively) expect that the direct inverse map should be able to predict these optimally projected Ludwik curves accurately, if provided with the bi-linear test points.

In this investigation we simulate the hoop strain-internal pressure responses for both the bi-linear and the optimally projected Ludwik material models, using FEA. These responses take on the role of virtual experimental data. These hoop strain-internal pressure responses are then mapped to the Ludwik material parameters, using the direct inverse map. The mapped parameters are given in Tab 1. The stress-strain responses are then computed by using the Ludwik material model parameters, which are shown with the actual bi-linear stress-strain responses in Fig. 8(a)-(b).

It is clear from Fig. 8 (a)-(b) that the direct inverse map is able to accurately predict the optimally projected Ludwik stress-strain response from the Ludwik-simulated hoop strain-internal pressure responses, because no model error is present in the simulated hoop strain-internal pressure responses. By contrast, the predicted stress-strain response obtained from the direct inverse mapped bi-linear simulated hoop strain-internal pressure response is significantly worse. We refer to this as a model mismatch resulting in a significant model error. This occurs because the bi-linear simulated hoop strain-internal pressure response cannot be described accurately by blending the simulated responses in the training set, since the training set uses the Ludwik material model.

The set of stress-strain curves that the Ludwik material model can represent is different from the set of stress-strain curves that the bi-linear material model can represent. This in turn significantly affects the set of hoop strain-internal pressure responses that can be computed from the two material models. For this reason, the hoop strain-internal pressure responses of the bi-linear and Ludwik models lie in two distinct subspaces in the 27 dimensional response space. Therefore, the bi-linear responses are outliers (different from the members of a specific space) to the Ludwik response subspace. Mapping a bi-linear generated hoop strain-internal pressure response with a direct inverse map which was constructed by using the Ludwik-simulated responses may therefore lead to poor predictions of the material parameters. It is essential to address this outlier response by ensuring that we only map the information in the bi-linear hoop strain-internal pressure responses that can be explained by the Ludwik-generated hoop strain-internal pressure responses in the training set. In this case it becomes essential that we project the bi-linear response onto the Ludwik subspace to first determine if the bi-linear response is an outlier among the Ludwik training responses. Next we consider some well-known projection strategies as well as the notion of outlier detection. These techniques are essential to determine whether the hoop strain-internal pressure response we intend to map can be explained by the training responses or whether the response is an outlier.

A common projection strategy is to decompose the hoop strain-internal pressure responses of the training data set into a basis or modes, using some basis extraction or data reduction technique such as principal component analysis (PCA) (Jolliffe 2002). Then we can project the hoop strain-internal pressure response of a test point onto the extracted basis. To this end, we express the test response by using the extracted modes of the training set in a least squares sense. We therefore approximate the test response by using the training responses. 
We first compute the principal directions of the training data set responses using PCA. The Ludwikgenerated training data set responses is then projected onto a selected number of principal directions. Then the direct inverse map is constructed, using the projected hoop strain-internal pressure responses of the training and validation data sets, as opposed to the original hoop straininternal pressure responses. The optimal range parameters for RBF are then computed as before.

Before mapping the bi-linear generated experimental response, we first project it onto the selected principal directions of the Ludwik-generated responses. As before, one RBF per material parameter is used in the direct inverse map. The obtained material parameters are then used to construct the material stress-strain curves, using the Ludwik material model. The authors refer to this technique as the RBFPC.

Fig. 9(a)-(d) depict the actual hoop strain-internal pressure responses for Test point 1 generated by the bi-linear material model. The figures also present the projected hoop strain-internal pressure response, using from one to four principal directions extracted from the training data generated by the Ludwik material model as well as the hoop strain-internal pressure response of the fitted Ludwik model. This gives a progressively improved description of the hoop strain-internal pressure response generated by using the bi-linear model, as the number of principal directions is increased. We need to exercise care when interpreting Fig. 9 because we are over-fitting the bi-linear generated material response when we use four principal directions to approximate the Ludwik responses in the training set. Although not shown, the Ludwik material model is not even able to generate an internal pressure-hoop strain response that is as accurate as the approximation using four principal directions.

The next step is to use the projected test points as input into the direct inverse map to provide the Ludwik material parameters. The results of this RBFPC procedure are shown in Fig. 10(a)-(b). For better clarity, the figures on the right only represent the stress-strain response of the actual bi-linear model in comparison with the stress-strain response obtained using RBFPC for one and two PCs. Here it can clearly be seen that using three principal directions results in inaccurate stress-strain responses, although the approximated hoop strain-internal pressure responses are nearly identical to the exact responses. It is clear that the greater the number of principal directions used, the better the description of the hoop strain-internal pressure response. We emphasise, however, that this does not imply a more accurate direct inverse map. This becomes clear when considering the principal components for each principal direction used to approximate the bi-linear generated hoop straininternal pressure response, in conjunction with the principal components of the training set.

We first project the hoop strain-internal pressure responses of the training set onto their principal directions to obtain the associated principal components (PCs) for each point in the training set. This gives us a good indication of the appropriate values of the principal components for each principal direction. Then we express the hoop strain-internal pressure responses of the test points, using the same principal directions. If the principal components for a principal direction in the projected subspace are significantly larger or smaller than the principal components of the training data set, we are extrapolating or dealing with an outlier in the projected space. Consequently, in such a case we are biasing our predictions with the associated risks of predicting a response which is isolated from the responses in the training dataset. We demonstrate this for the two bi-linear material model hoop strain-internal pressure curves in Fig. 11(a)-(d), using from one to four principal components in the box plots respectively .

The box-whisker plot indicates the principal components for the training dataset, where the blue box indicates the lower and upper quartile values, the red line indicates the median, and the whiskers indicate the extent of the remainder of the data. The principal components for the bi-linear generated hoop strain-internal pressure curves are indicated by the annotations. It is clear from Fig. 
11(a) that the principal components of the test points for the first principal direction lie within the range of the principal components of the training data. Fig. 11(b) indicates that the principal components of the test points for the second principal direction lie just outside the range of the principal components of the training data. In this case, we are running the risk of generating inaccurate mappings. It is clear from Fig. 11(c) that the principal components for the third principal direction of both test points lie far beyond the principal component ranges of the points in the training set. Fig. 11(d) indicates that the fourth principal component of the first example falls within the training set range but each mode contributes progressively less information to the total information contained in the dataset. Fig. 11 clearly indicates that the contributions of the principal directions required obtaining accurate hoop strain-internal pressure curves are not present in the Ludwik-generated responses in the training set. Hence the poor stress-strain curves generated from the mapped Ludwik material parameters come as no surprise. In other words, we need to take care that we only map those parts of the bi-linear generated hoop strain-internal pressure response that the Ludwik-generated hoop strain-internal pressure responses in the training set can represent. This also implies that we should not trust the predicted material parameters that follow from test responses which are isolated from the training data responses. Hence, projection plays an essential role in the direct inverse map.

It would be convenient if we could avoid finding the optimal range parameter that is so important for the RBF-based direct inverse map. We investigate if a simple linear regression is sufficient as opposed to a non-linear blending. In this case, it was found to be adequate for both test points. The linear regression is conducted in the form of principal component regression (PCR) (AndradeGarda 2009a), where we map the principal components with a linear regression to the Ludwik material parameters. The benefit of the linear regression is that we do not have to estimate an optimal range parameter. Fig. 12 demonstrates the similar performance between the RBFPC and PCR, indicating that a linear blending between the principal components to map to the material model parameters is sufficient for predicting the stress-strain behaviour of Test point 1 . The setup time and computational cost when opting for PCR are reduced and the benefits are clear.

\section{The ability of PLSR to handle stochastic noise in the system response}

The effectiveness of PLSR to handle stochastic noise with a zero mean is considered, using the problem with $\pm 2 \%$ uniform stochastic noise added to the hoop strain-internal pressure responses of the test sets. In order to use PLSR we need to select the proper number of scores (loadings). Fig. 13 depicts the accuracy of the PLSR approach to predict the stress-strain response using one, three and five scores respectively. Fig. 13 clearly demonstrates an increase in accuracy with an increase in the number of scores. Note, however, that for one and three scores the same accuracy is obtained for noiseless and $\pm 2 \%$ uniform stochastic noise added to the hoop strain-internal pressure test sets. It is clear that when five scores are considered, there is a disparity between the stress-strain responses of the noiseless and the $\pm 2 \%$ uniform stochastic noise test sets, indicating that five scores is overfitting the Ludwik material model. In addition, we know that the Ludwik model has only three parameters, so we expect that three scores should capture the behaviour of the internal pressure-hoop strain response and the Ludwik material parameter data.

An additional computational benefit of the PLSR approach is that it allows us to map directly from the hoop strain-internal pressure curve to the Ludwik stress-strain curve. This would be impractical for the RBFPC approach as each point on the stress-strain curve would have to be computed by means of a different RBF. We repeat the previous experiment with $\pm 2 \%$ uniform stochastic noise added to the hoop strain-internal pressure test data, but in this case we map directly to the Ludwik stress-strain curves as opposed to the Ludwik material parameters. Fig. 14 depicts a significant increase in accuracy when considering high dimensional data for the system response and for the 
system parameters. This increased accuracy probably reflects a more linear relationship between the measured response and the stress-strain curve, as compared to the relationship between the measured response and the material parameters. Similarly to Fig. 13, the use of one and three scores have essentially the same response for the noiseless and the $\pm 2 \%$ uniform stochastic noise test sets, whereas with five scores the results differ significantly. This suggests a pragmatic strategy to select the number of scores. As expected, PLSR with three scores is more likely to better predict the stress-strain curve from hoop strain-internal pressure data with $\pm 2 \%$ stochastic noise than the PLSR with one or five scores.

\section{The ability of partial least squares regression to address model mismatch}

Next we repeat the numerical experiment for the model mismatch experiment, using PLSR. Recall that the hoop strain-internal pressure responses for the two test points are generated using the bilinear material model and the training set responses are generated using the Ludwik model.

Fig. 15(a)-(b) depict the stress-strain responses by directly mapping the hoop strain-internal pressure testing data using partial least squares regression for the two bi-linear system responses. As before, it is clear from Fig. 15(a)-(b) that the number of scores significantly affects the ability of PLSR to map the hoop strain-internal pressure responses directly to the stress-strain responses. For both test points, PLSR with one and two scores perform well. However, using the third score significantly degrades the predicted stress-strain curves.

To quantify whether the bi-linear response is an outlier in the projected Ludwik response space, we first project the training set hoop strain-internal pressure curves onto the PLSR training set loadings to obtain the associated scores (principal components) for each point in the training set. These scores are depicted in Fig. 16(a)-(c). We then express the scores of the two bi-linear test points in the space of training set loadings, also included in Fig. 16(a)-(c). It is clear from Fig. 16(a) that the first projected scores (test scores) lie within the range of the training scores. We note from Fig. 16(b) that both test point scores lie outside the range of the training scores (outliers). In this instance, we are running the risk of generating inaccurate mappings. It is clear from Fig. 16(c) that the scores of both test points lie far beyond the range of the training data scores.

Similar to PCR, the only parameter that PLSR requires is the number of scores. A pragmatic guide to selecting the number of scores is to ensure that we are not obviously predicting outlier responses in the projected score subspace of the training dataset. Formal outlier detection strategies can, however, significantly refine and improve this observation (Jolliffe 2002). Alternatively, a view can be taken where the confidence in the mapping is related to the density of the surrounding points in the score subspace. The benefit of PLSR is that it is computationally efficient and numerically convenient as it is available in many numerical packages, e.g. Matlab, R, and programming libraries e.g. Python SciPy. This would allow the analysts to make quick progress towards solving and understanding the inverse problem.

\subsection{Recovering material model parameters from experimental CP-Titanium}

The tube bulge test example only used simulated data (for the training, validation and test points), to allow explicit control over the types and magnitude of noise. In our second example we identify material parameters from published experimental stress-strain data for commercially pure (Roemer et al.) Titanium. We select the experiment at a temperature of $373 \mathrm{~K}$ and a strain rate of $0.001 \mathrm{~s}^{-1}$ from the paper by (Nemat-Nasser et al. 1999), and tabulate our digitized values in Tab 2. It is clear that at these experimental conditions CP-Titanium has a non-conventional stress-strain response as the strain hardening rate does not decrease monotonically. 
We consider the well-known state based Voce strain-hardening model for which we aim to estimate the material parameters. The Voce strain-hardening model is stated in differential form as

$$
\frac{d \sigma}{d \varepsilon}=\theta_{0}\left(1-\frac{\sigma}{\sigma_{s}}\right)
$$

with initial condition $\left.\sigma\right|_{\varepsilon=0}=\sigma_{0}$. The model parameters are the initial hardening rate $\left(\theta_{0}\right)$, the saturation stress $\left(\sigma_{s}\right)$ and the initial yield stress $\left(\sigma_{0}\right)$.

The purpose of this example is to provide a benchmark problem that does not require significant setup time. The simulation model in this case is a simple numerical integrator, but this does not detract from the validity of this numerical example. This example allows familiarisation of the techniques discussed and independent verification by other researchers.

We attempt to recover the experimental stress-strain curve by directly mapping the stress-strain curve to the Voce model parameters. Only 30 simulated curves are used in our training set using the parameter ranges in Tab 3. We consider regression RBF, RBFPC and PLSR with 1 to 4 modes to reconstruct the measured response using the Voce model. This example again emphasises the importance of projecting the measured response onto the simulated response space.

The stress-strain curves computed from the mapped Voce model material parameters are depicted in Fig. 17 and Fig. 18 for the RBF and PLSR based approaches respectively. These curves are compared to the experimentally measured curve and the best fitted (least squares optimisation) curve. The corresponding parameters are also represented in Tab 4. Notice that the best fitted curve results in an error (norm of the difference between two curves) of 46.5, while the RBF direct inverse map and PLSR method provides errors of 46.6 and 46.9 respectively. This indicates that the direct inverse map approaches are both able to find good approximations to the solution, without the need to perform optimisation. It is also clear from Fig. 17 that projection is beneficial to accurately represent the experimental stress-strain curve as the regression RBF with $2 \%$ noise fails to accurately capture the experimental stress-strain curve. We include the principal components / scores boxplots of the projected training data points and the projected measured stress-strain curve in Fig. 17 and Fig. 18. This allows us to observe the degree of extrapolation as the number of modes increases. For the first three modes we note that the principal components / scores of the projected experimental stress-strain curve lie within the boxplot whiskers. As before, this enhances our confidence that we are not extrapolating out of the training data set. This is in contrast to the fourth mode for which the principal component / score of the projected experimental stress-strain curve are orders larger than the depicted range. The fourth PC of the projected experiment is 40.9 and the fourth PLSR score is -41 . The quality of the stress-strain curves computed from the mapped Voce model parameters using 1 to 3 modes is evident when comparing to the best fitted Voce model. The stress-strain curves obtained from the mapped Voce model parameters using four principal components / scores, however do not even fit within the figure bounds. Note that all these results were obtained without the need for optimization and we only required 30 computed stress-strain curves using the Voce model for the training data set.

\section{Conclusion}

This paper demonstrates that the direct inverse map approach is viable for finding quick solutions to material identification problems, but care and consideration should be taken when constructing the direct inverse map. The presence of stochastic noise in the experimental results as well as the potential mismatch between the simulation model and experiment may yield poor results. The importance of projection to address model error was demonstrated on simulated experimental data 
and actual experimental data using RBFPC and PLSR. We noted that PLSR performed comparable to RBFPC on all problems considered. This makes partial least squares regression an attractive starting point for constructing direct inverse maps, as it addresses the issues of stochastic noise and model mismatch, while the only parameter is the number of principal components /scores that should be considered. A pragmatic guide to selecting the number of principal components / scores is to ensure that there is no obvious extrapolation in the scores subspace of the training dataset. The additional benefit of PLSR is that it is computationally efficient and available in many numerical packages, allowing the analyst to make quick progress with understanding and solving the inverse problem.

\section{Acknowledgment}

The authors would like to acknowledge the National Research Foundation (NRF), the Technology and Human Resources for Industry Programme (THRIP), and the Eskom Power Plant Engineering Institute (EPPEI) for the support given to make the research possible. We also would like to thank the reviewers for their suggestions that enhanced the clarity and enriched the contribution of this study.

\section{References}

Abbassi F, Belhadj T, Mistou S, Zghal A (2013) Parameter identification of a mechanical ductile damage using Artificial Neural Networks in sheet metal forming. Mater Des. 45:605-615 doi:/10.1016/j.matdes.2012.09.032

Aguir H, BelHadjSalah H, Hambli R (2011) Parameter identification of an elasto-plastic behaviour using artificial neural networks-genetic algorithm method. Mater Des. 32:48-53

Aguir H, Chamekh A, BelHadjSalah H, Dogui A, Hambli R (2008) Identification of constitutive parameters using hybrid ANN multi-objective optimization procedure. Int J Mater Form. 1:1-4

An G (1996) The Effects of Adding Noise during Backpropagation Training on a Generalization Performance. Neural Comput. 8:643-674

Andrade-Garda JM (2009a) 3. Ordinary Multiple Linear Regression and Principal Components Regression. In: Basic Chemometric Techniques in Atomic Spectroscopy. Royal Society of Chemistry.

Andrade-Garda JM (2009b) 4. Partial Least-Squares Regression. In: Basic Chemometric Techniques in Atomic Spectroscopy. Royal Society of Chemistry.

ANSYS $^{\circledR}$ Academic Research, Release 14,5.

Asaadi E, Heyns P (2016) Flow stress identification of tubular materials using the progressive inverse identification method. ENG COMPUTATION. 33

Bolzon G, Talassi M (2013) An effective inverse analysis tool for parameter identification of anisotropic material models. Int J Mech Sci. 77:130-144

Bortot P, Ceretti E, Giardini C (2008) The determination of flow stress of tubular material for hydroforming applications. J Mater Process Technol. 203:381-388

Brigham JC, Aquino W (2007) Surrogate-Model Accelerated Random Search algorithm for global optimization with applications to inverse material identification. COMPUT METHOD APPL M. 196:4561-4576 
Brown WM, Gedeon TD, Groves DI (2003) Use of noise to augment training data: A neural network method of mineral-potential mapping in regions of limited known deposit examples. Nat Resour Res. 12:141-152

Buhmann MD (2004) Radial Basis Functions. theory and implementation CAMBRIDGE UNIVERSITY PRESS.

Chamekh ABHSHHR (2009) Inverse technique identification of material parameters using finite element and neural network computation. Int J Adv Manuf Technol. 44:1-2

Chen S (2009) Another Particle Swarm Toolbox. http://www.mathworks.com/matlabcentral/fileexchange/25986-another-particle-swarm-toolbox. Accessed 2014-03-10

Degroote J, Couckuyt I, Vierendeels J, Segers P, Dhaene T (2012) Inverse modelling of an aneurysm's stiffness using surrogate-based optimization and fluid-structure interaction simulations. Struct Multidisc Optim. 46:457-469 doi:10.1007/s00158-011-0751-7

Fasshauer G, Zhang J (2007) On choosing "optimal" shape parameters for RBF approximation. Numer Algor. 45:345-368 doi:10.1007/s11075-007-9072-8

Gogu C, Passieux JC (2013) Efficient surrogate construction by combining response surface methodology and reduced order modeling. Struct Multidisc Optim. 47:821-837 doi:10.1007/s00158-012-0859-4

Hambli R, Guerin F (2003) Application of a neural network for optimum clearance prediction in sheet metal blanking processes. Finite Elem Anal Des. 39:1039-1052

Harb N, Labed N, Domaszewski M, Peyraut F (2014) Optimization of material parameter identification in biomechanics. Struct Multidisc Optim. 49:337-349 doi:10.1007/s00158-013-0973-y

Hastie T, Tibshirani R, Friedman J (2003) The Elements of Statistical Learning: Data Mining, Inference, and Prediction. The Elements of Statistical Learning. Springer. doi:citeulike-article-id:161814

Huber N, Tsakmakis C (2001) A neural network tool for identifying the material parameters of a finite deformation viscoplasticity model with static recovery. COMPUT METHOD APPL M. 191:353-384

Jolliffe IT (2002) Principal component analysis. Springer-Verlag.

Kaspar MH, Ray WH (1993) Partial least squares modelling as successive singular value decompositions. Comput Chem Eng. 17:985-989

Lianfa Y, Cheng G (2008) Determination of stress-strain relationship of tubular material with hydraulic bulge test. Thin Walled Struct. 46:147-154

Matlab R2015a. Natick, Massachusetts. The Mathworks Inc, (2015)

Munoz-Sánchez A, González-Farias IM, Soldani X, Miguélez MH (2011) Hybrid FE/ANN and LPR approach for the inverse identification of material parameters from cutting tests. Int J Adv Manuf Technol. 54:21-33

Nemat-Nasser S, Guo WG, Cheng JY (1999) Mechanical properties and deformation mechanisms of a commercially pure titanium. 47:3705-3720 doi:10.1016/\$1359-6454(99)00203-7

Oden JT, Prudhomme S (2002) Estimation of modeling error in computational mechanics. J Comput Phys. 182:496-515 doi:10.1006/jcph.2002.7183 
Oliver DS, Reynolds AC, Liu N (2008) 3. Estimation for Linear Inverse Problems. In: Inverse Theory for Petroleum Reservoir Characterization and History Matching. Cambridge University Press.

R: A Language and Environment for Statistical Computing 3.1.2 R Foundation for Statistical Computing, (2014)

Roemer MJ, Kacprzynski GJ, Schoeller MH Improved diagnostic and prognostic assessments using health management information fusion. In: AUTOTESTCON (Proceedings), 2001. pp 365-377

Song H, Hashash YMA (2015) Characterization of stress-strain behaviour of red blood cells (RBCs), part I: methodology development and application to healthy RBCs. Inverse Prob Sci Eng. 23:913-931 doi:10.1080/17415977.2014.954111

Stavroulakis GE, Bolzon G, Waszczyszyn Z, Ziemianski L (2003) 3.13 - Inverse Analysis. In: Milne I, Ritchie RO, Karihaloo B (eds) Comprehensive Structural Integrity. Pergamon, Oxford, pp 685-718. doi:/10.1016/B0-08-043749-4/03117-7

Sun D-W (2008) 3. Multivariate Calibration for Quantitative Analysis. In: Infrared Spectroscopy for Food Quality Analysis and Control. Elsevier.

Tikhonov ANA, Vasiliy Y. (1977) Solutions of III-posed Problems John Wiley \& Sons Inc.

SciPy: Open source scientific tools for Python 0.14.0. Community library project, (2014)

Uhl T (2007) The inverse identification problem and its technical application. Arch Appl Mech. 77:325-337 doi:10.1007/s00419-006-0086-9

Xu Y, Chan LC, Tsien YC, Gao L, Zheng PF (2008) Prediction of work-hardening coefficient and exponential by adaptive inverse finite element method for tubular material. J Mater Process Technol. 201:413-418

Zhang S, Leotoing L, Guines D, Thuillier S, Zang S-I (2014) Calibration of anisotropic yield criterion with conventional tests or biaxial test. Int J Mech Sci. 85:142-151 doi:dx.doi.org/10.1016/j.ijmecsci.2014.05.020

Zhang Y, Sun G, Xu X, Li G, Huang X, Shen J, Li Q (2013) Identification of material parameters for aluminum foam at high strain rate. Int J Comput Mater Sci. 74:65-74

Zribi T, Khalfallah A, BelHadjSalah H (2013) Experimental characterization and inverse constitutive parameters identification of tubular materials for tube hydroforming process. Mater Des. 49:866877 doi:/10.1016/j.matdes.2013.02.077 
Tab. 1 Test points chosen for the bi-linear model, and the corresponding optimally projected and mapped Ludwik models

\begin{tabular}{|l|c|c|}
\hline Material model & Test point 1 & Test point 2 \\
\hline Bi-linear model & $\sigma=200+2600 \varepsilon$ if $\varepsilon \leq 0.09$ & $\sigma=210+3300 \varepsilon$ if $\varepsilon \leq 0.07$ \\
\hline $\begin{array}{l}\text { Optimally projected } \\
\text { Ludwik model }\end{array}$ & $\sigma=434+1100(\varepsilon-0.09)$ if $\varepsilon>0.09$ & $\sigma=441+900(\varepsilon-0.07)$ if $\varepsilon>0.07$ \\
\hline $\begin{array}{l}\text { Predicted Ludwik model by } \\
\text { mapping the optimally } \\
\text { projected Ludwik- } \\
\text { generated hoop strain- } \\
\text { pressure response. }\end{array}$ & $\sigma=181+1092 \varepsilon^{0.64}$ & $\sigma=193+976 \varepsilon^{0.56}$ \\
\hline $\begin{array}{l}\text { Predicted Ludwik model by } \\
\text { mapping the bi-linear } \\
\text { generated hoop strain- } \\
\text { pressure response. }\end{array}$ & $\sigma=177+1092 \varepsilon^{0.63}$ & $\sigma=192+934 \varepsilon^{0.54}$ \\
\hline
\end{tabular}

Tab 2 CP-titanium stress-strain data

\begin{tabular}{|c|c|c|c|c|c|c|c|c|c|c|}
\hline $\begin{array}{c}\text { Plastic } \\
\text { strain }\end{array}$ & 0.02 & 0.04 & 0.06 & 0.08 & 0.10 & 0.12 & 0.14 & 0.16 & 0.18 & 0.20 \\
\hline $\begin{array}{c}\text { Stress } \\
\text { (MPa) }\end{array}$ & 149 & 181 & 202 & 223 & 245 & 272 & 302 & 336 & 366 & 391 \\
\hline $\begin{array}{c}\text { Plastic } \\
\text { strain }\end{array}$ & 0.22 & 0.24 & 0.26 & 0.28 & 0.30 & 0.32 & 0.34 & 0.36 & 0.38 & 0.40 \\
\hline $\begin{array}{c}\text { Stress } \\
\text { (MPa) }\end{array}$ & 410 & 428 & 442 & 454 & 465 & 474 & 484 & 490 & 498 & 507 \\
\hline
\end{tabular}

Tab 3 Range of the parameters used to constructed the training set

\begin{tabular}{|l|l|}
\hline Parameter & Range \\
\hline$\theta_{0}$ & $1500-2500$ \\
\hline$\sigma_{y s}(\mathrm{MPa})$ & $400-700$ \\
\hline$\sigma_{y 0}(\mathrm{MPa})$ & $100-200$ \\
\hline
\end{tabular}


Tab 4 Parameters of the Voce model computed with least square, RBF and PLSR based approaches with different number of principal component / score

\begin{tabular}{|c|c|c|c|c|c|c|c|c|c|c|}
\hline \multirow{2}{*}{ Parameter } & $\begin{array}{c}\text { best fitted } \\
\text { Voce } \\
\text { model }\end{array}$ & \multicolumn{5}{|c|}{ RBF } & \multicolumn{4}{c|}{ PLSR } \\
\cline { 3 - 12 } & Regression & 1 PC & 2 PCs & 3 PCs & 4 PCs & 1 score & 2 scores & 3 scores & 4 scores \\
\hline$\theta_{0}$ & 1850 & 1442 & 2115 & 2007 & 1820 & 758 & 2120 & 2056 & 1812 & 793 \\
\hline$\sigma_{y s}(\mathrm{MPa})$ & 509 & 494 & 492 & 507 & 517 & 40 & 490 & 524 & 515 & -2.58 \\
\hline$\sigma_{y 0}(\mathrm{MPa})$ & 135 & 153 & 122 & 127 & 136 & 154 & 121 & 116 & 136 & 154 \\
\hline error & 46.5 & 118.5 & 54.4 & 52.4 & 46.6 & 928.6 & 53.4 & 57.2 & 46.9 & NA \\
\hline
\end{tabular}

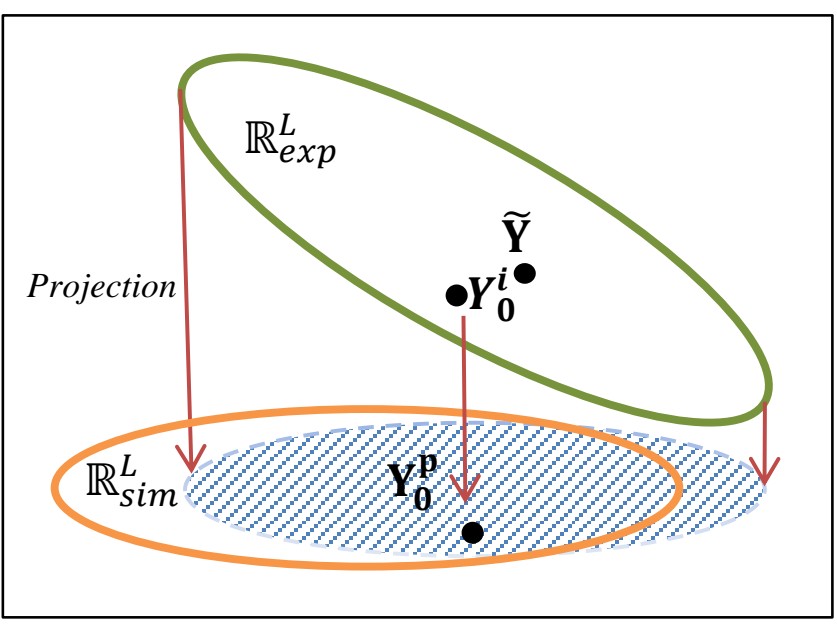

Fig. 1 Pictorial representation of the relationship between the experimental response space and the simulation response space 

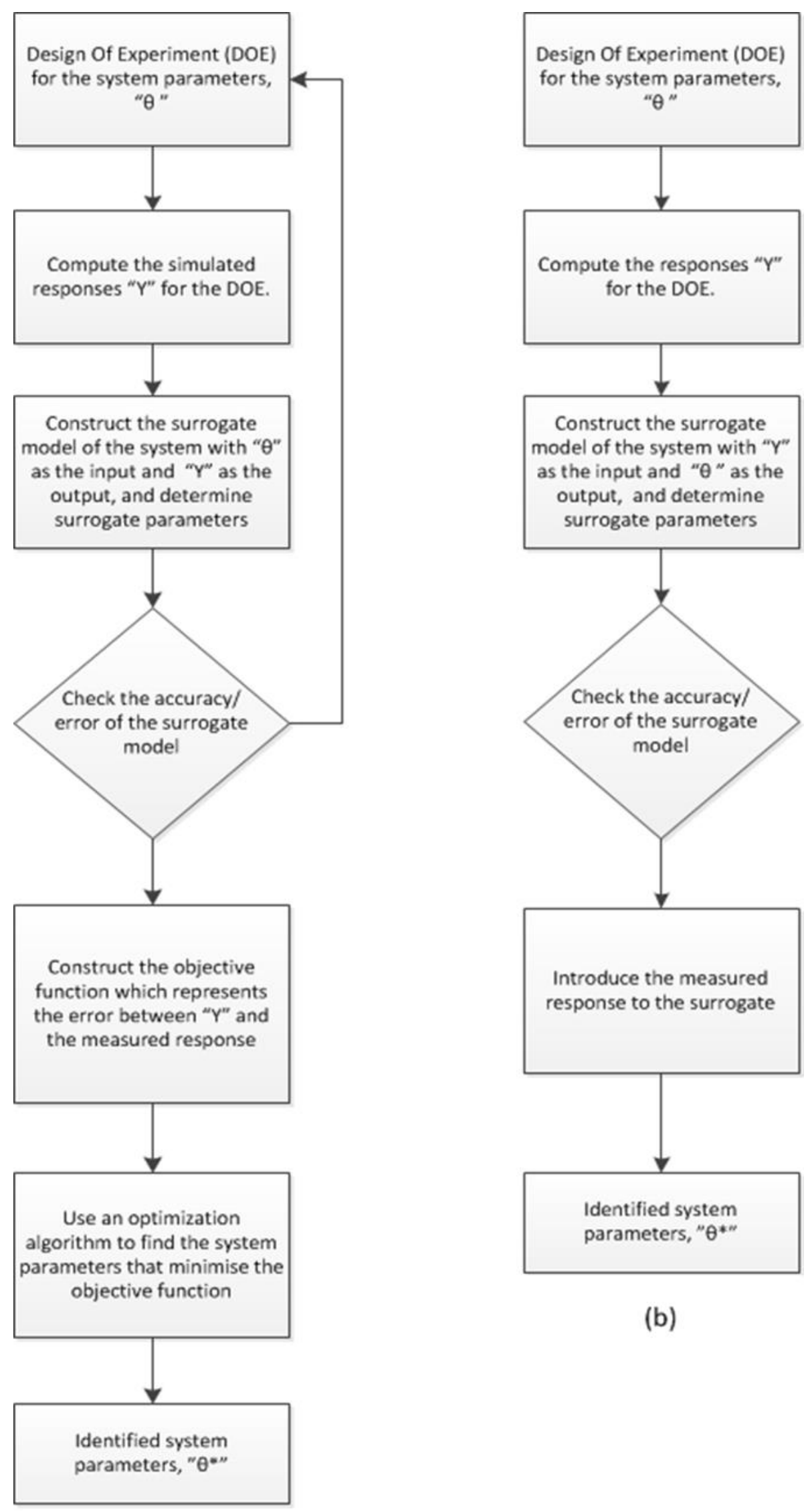

(b)

(a)

Fig. 2 Typical procedures for (a) the forward inverse identification method, and (b) the backward inverse identification method (direct inverse map) 


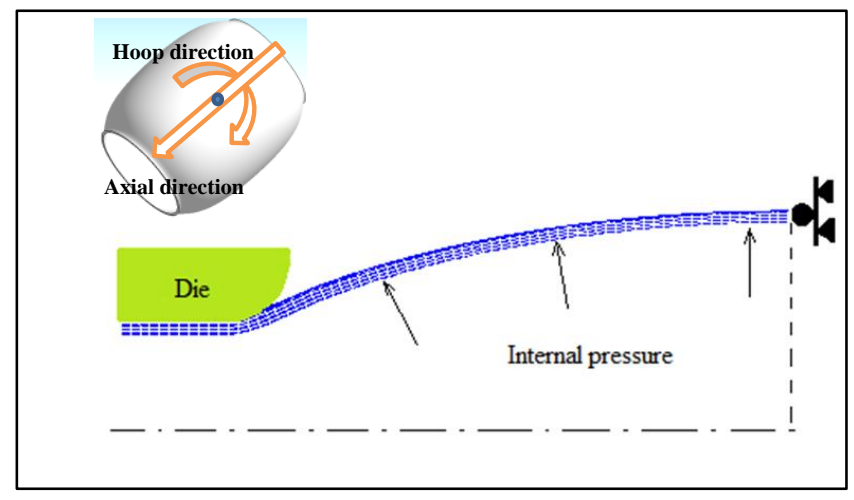

Fig. 3 Axisymmetric finite element model of the tube bulge test with reflection symmetry across the vertical axis

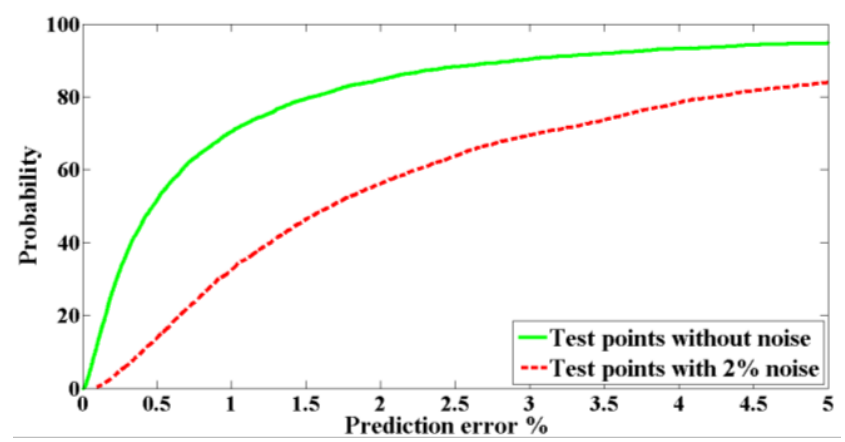

Fig. 4 Probability that the direct inverse map predicts the stress-strain response below a specific percentage error, when the test points are noiseless (solid green) and noisy (dashed red)

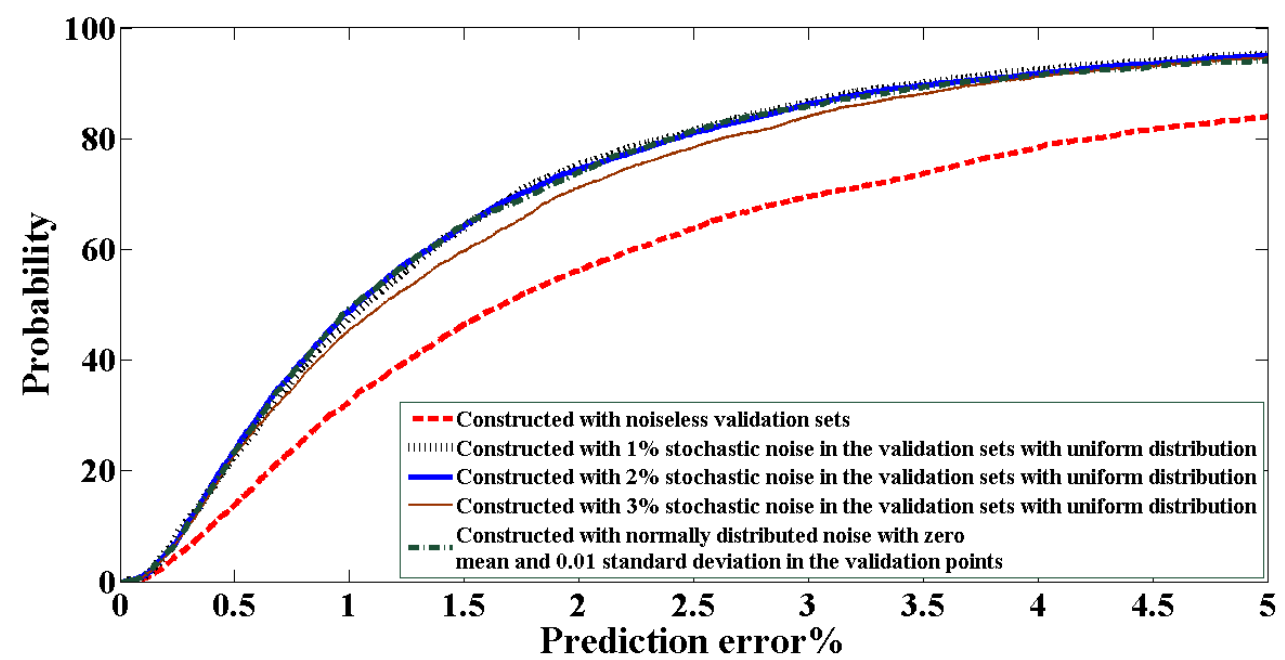

Fig. 5 Probability that the direct inverse map predicts the stress-strain response below a specific percentage error, when $2 \%$ stochastic noise is added to the test points. The optimal range parameters for the direct inverse maps are computed for the repeated validation points in the validation set with various distributions and magnitude of stochastic noFeise added 


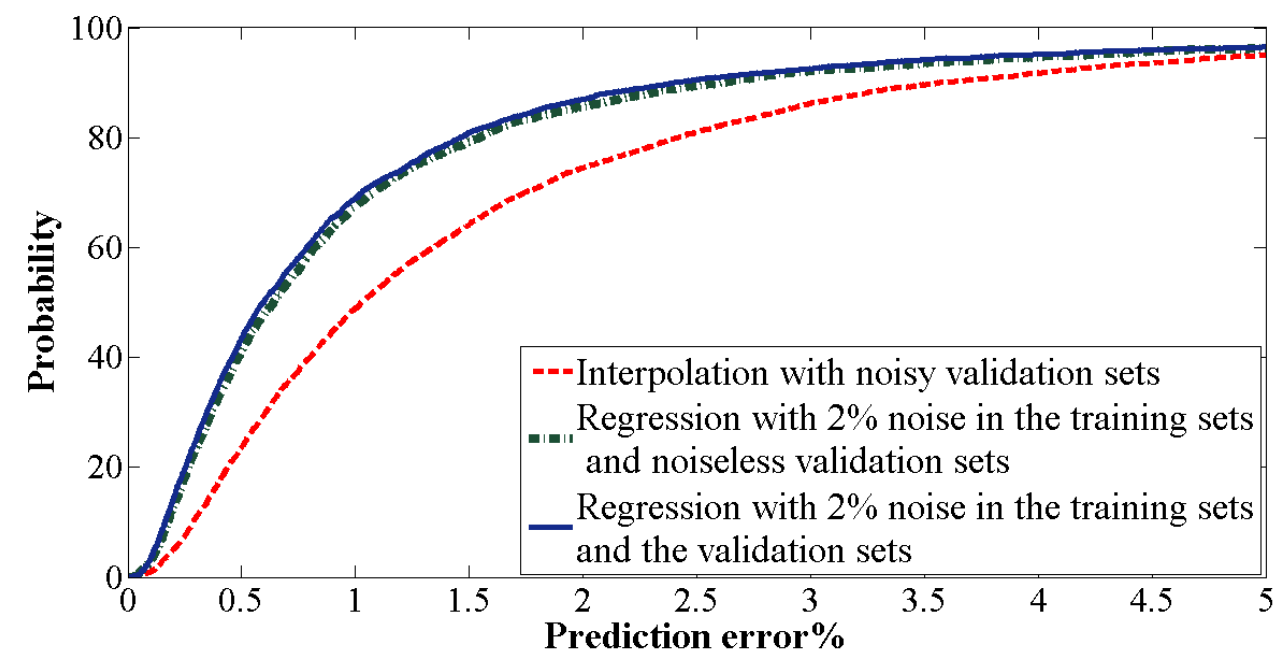

Fig. 6 Probability that the direct inverse map predicts the stress-strain response below a specific percentage error, when constructing the RBF by regression as opposed to interpolation. In order to construct the regression RBF, the training points with $2 \%$ stochastic noise in the simulated responses are repeated in the training set

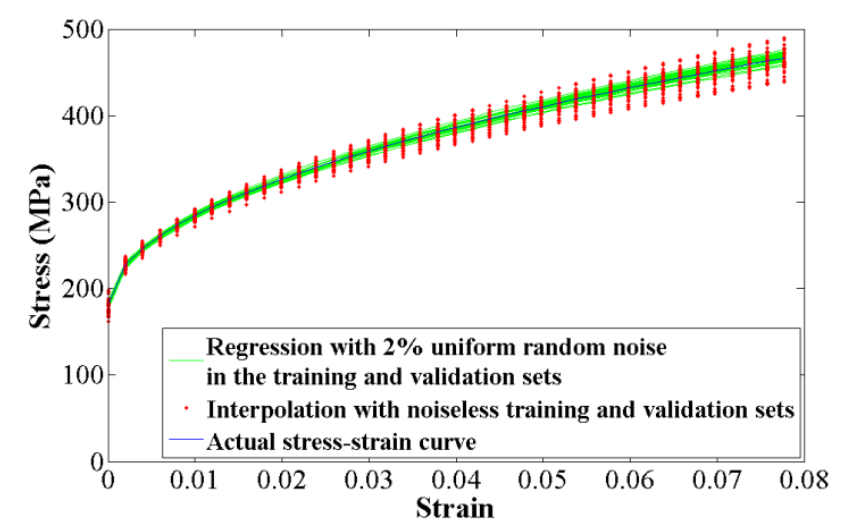

Fig. 7 Predicting a stress-strain curve when $2 \%$ uniform random noise is added to the test point and the mapping model is i) interpolation with noiseless validation sets or ii) regression with $2 \%$ uniform random noise added to the validation and training sets

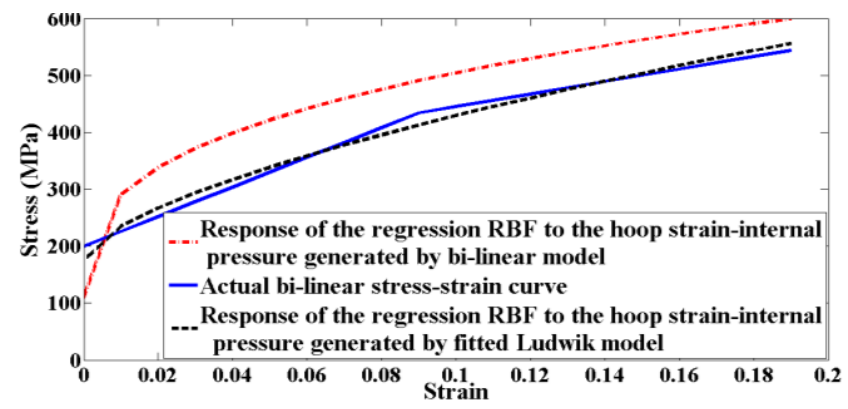

(a)

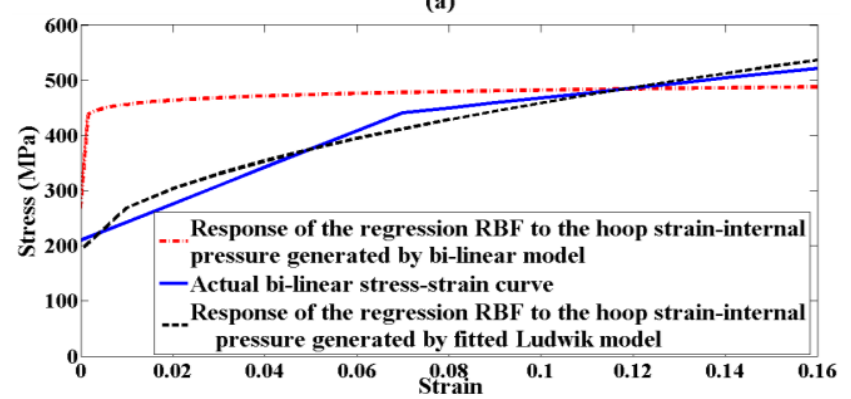

(b)

Fig. 8 Responses of the inverse mapping when the hoop strain-internal pressure data generated with the bi-linear model (red dashed 
lines), hoop strain-internal pressure data generated by the best projected Ludwik model, and the actual bi-linear stress-strain curves for (a) Test point 1 and (b) Test point 2

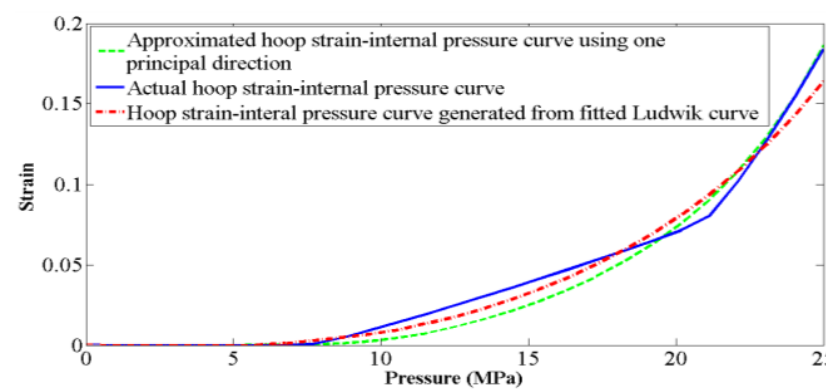

(a)

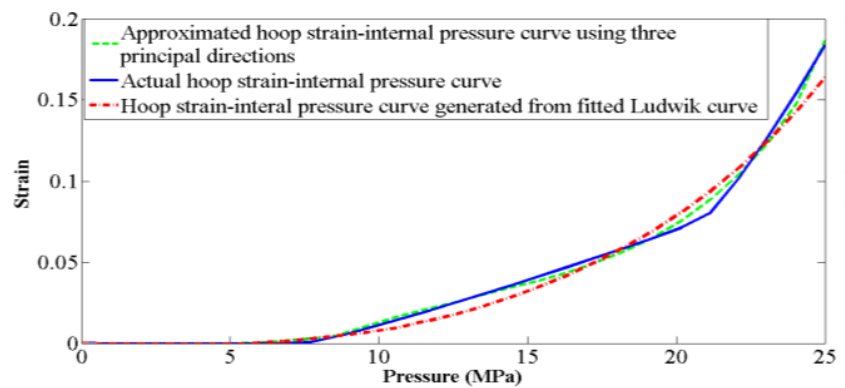

(c)

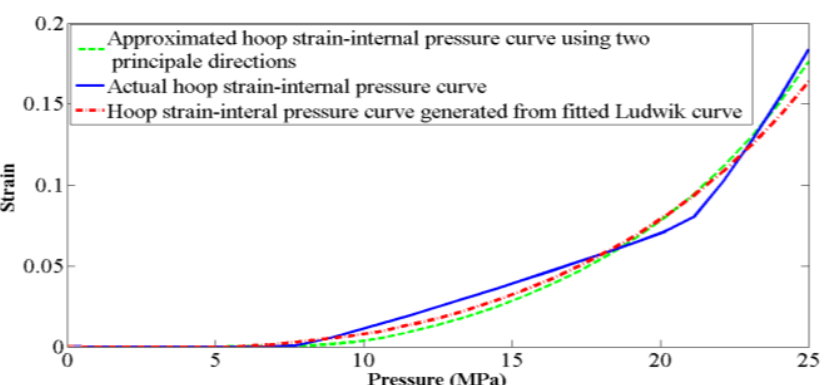

(b)

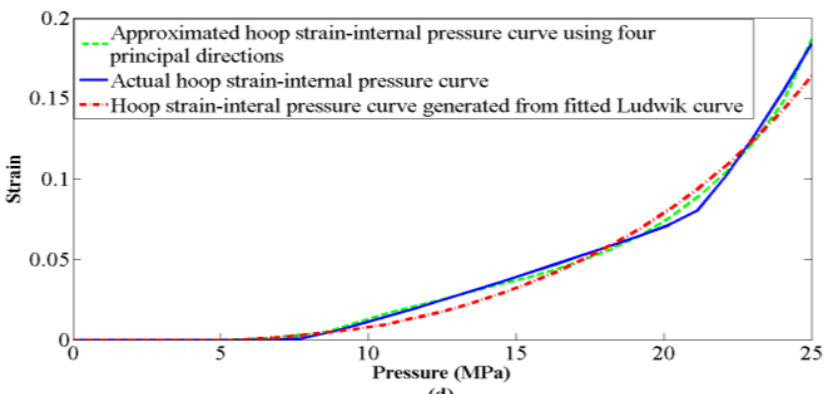

(d)

Fig. 9 The bi-linear material model-generated hoop strain-internal pressure curve of Test 1, and the least-squares projection using (a) one (b) two (c) three and (d) four principal directions extracted from the Ludwik material model-generated training data

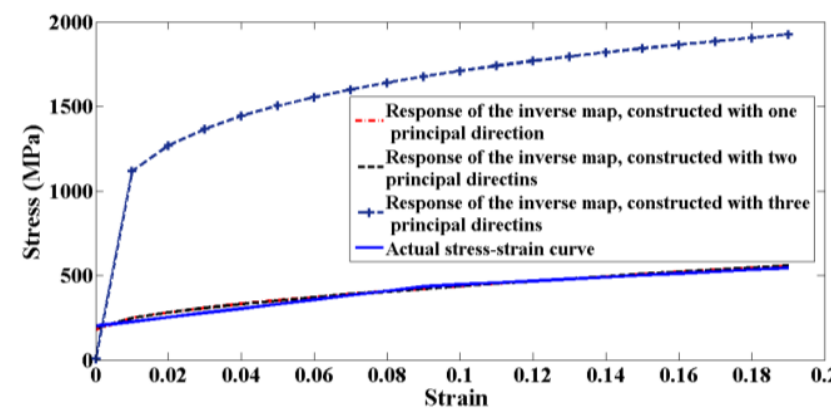

(a)
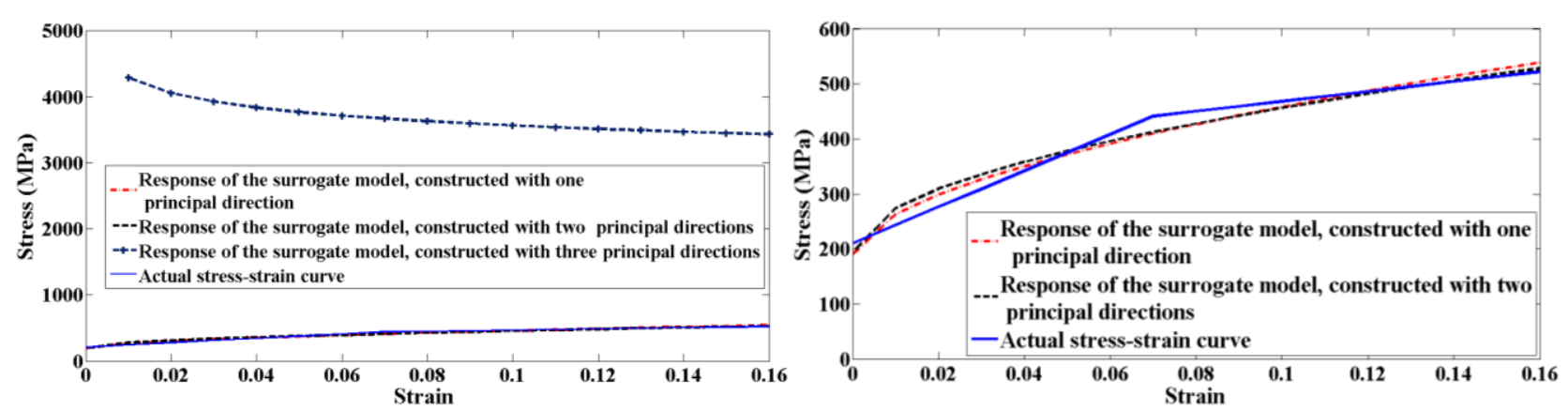

(b)

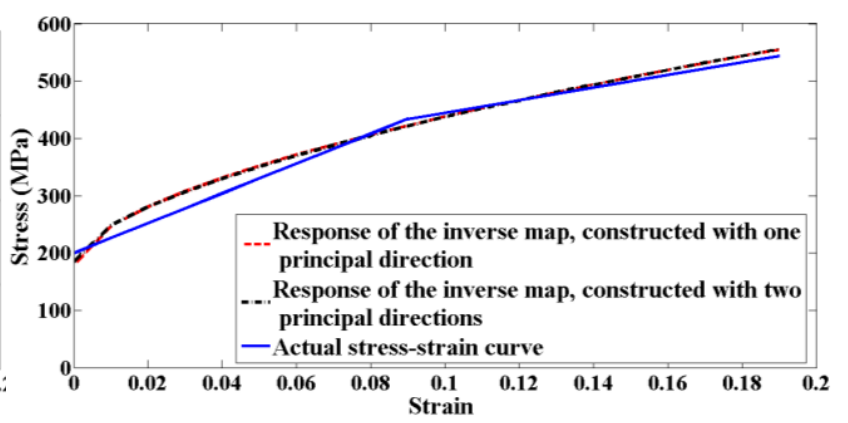

Fig. 10 Stress-strain curves generated from the Ludwik material parameters obtained by the direct inverse map of the approximated hoop strain-internal pressure curves for (a) Test point 1 and (b) Test point 2 . For better clarity, the figures on the right just represent the stress-strain response of the actual bi-linear model in comparison with the stress-strain response obtained using RBFPC for one and two PCs. 

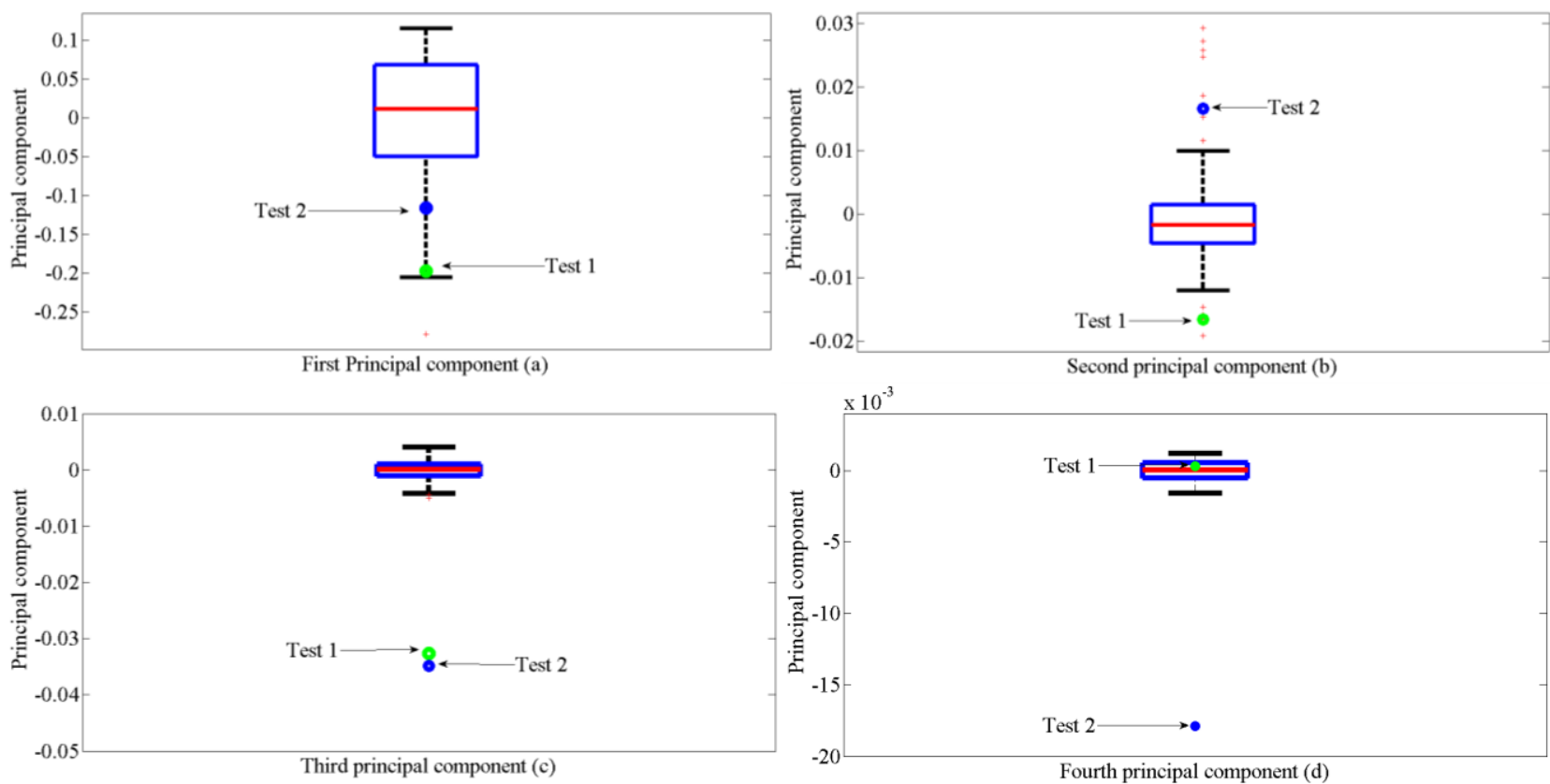

Fig. 11 Box plots of the principal components to describe the hoop strain-internal pressure of the training data generated by using the Ludwik material model and the test data generated by two bi-linear material models for (a) the first, (b) second, (c) third and (d) fourth principal direction

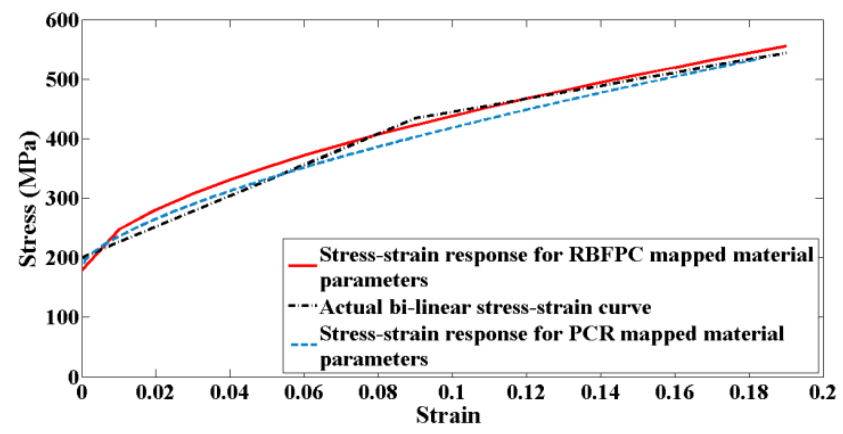

Fig. 12 Comparison between the stress-strain response for the first test point when mapping the principal components of the system response to the Ludwik material parameters linearly using PCR, and non-linearly using RBFPC

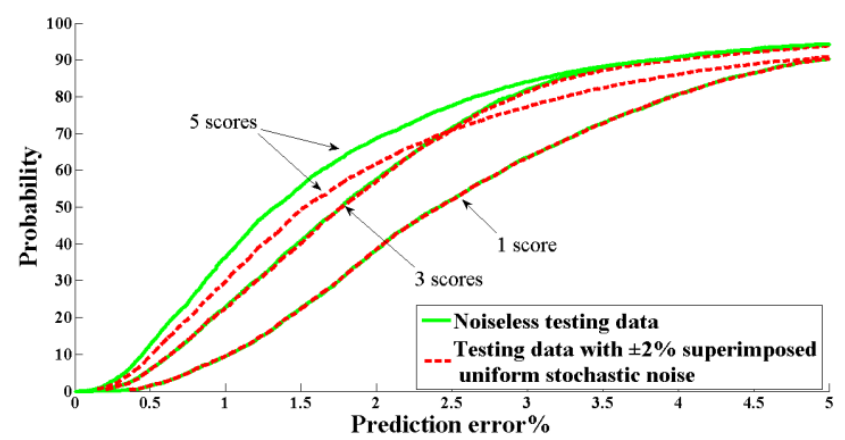

Fig. 13 Probability that PLSR predicts a stress-strain curve below a specified percentage error using 1,3 and 5 scores with $\pm 2 \%$ uniform stochastic noise added to the hoop strain-internal pressure response of the test points 


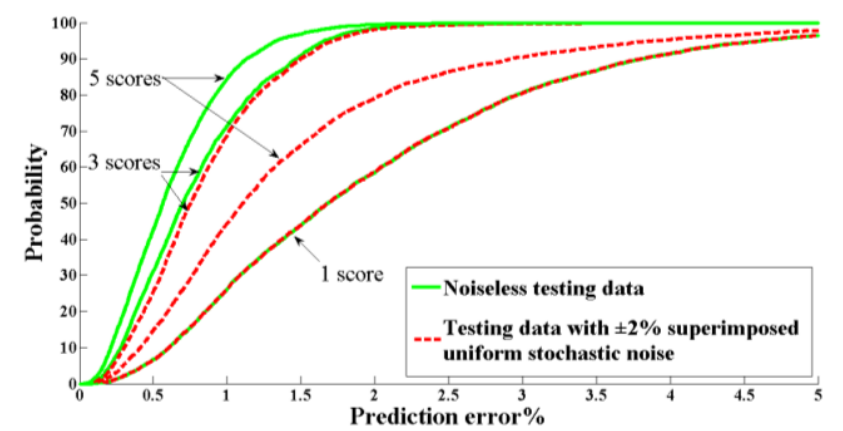

Fig. 14 Probability that PLSR predict stress-strain curves below a specified percentage error when the mapping is from the hoop strain-internal pressure data with $\pm 2 \%$ uniform stochastic noise to the stress-strain curves, using 1,3 and 5 scores

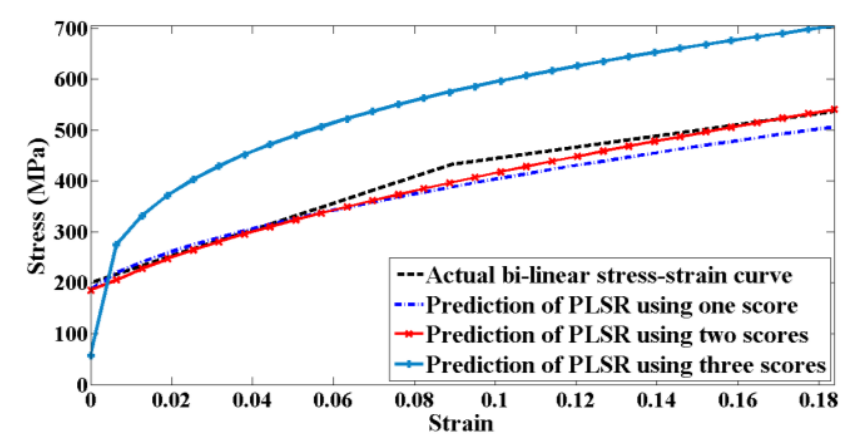

(a)

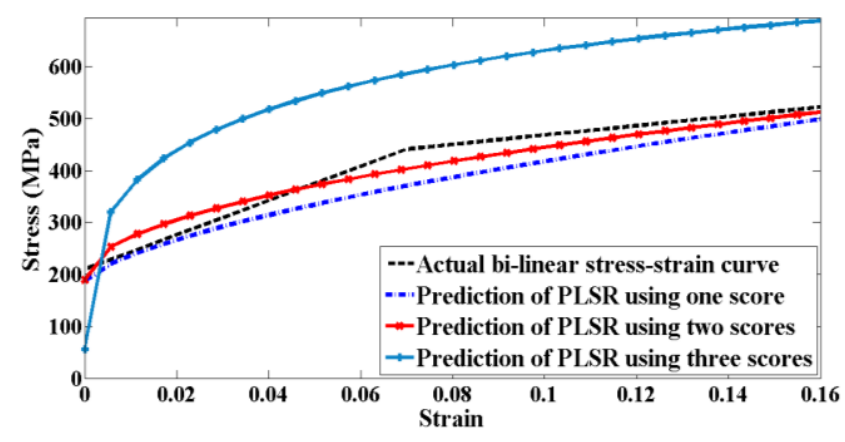

(b)

Fig. 15 Stress-strain curves obtained by directly mapping the hoop strain-internal pressure curves for (a) Test point 1 and (b) Test point 2, using partial least squares regression (PLSR) 

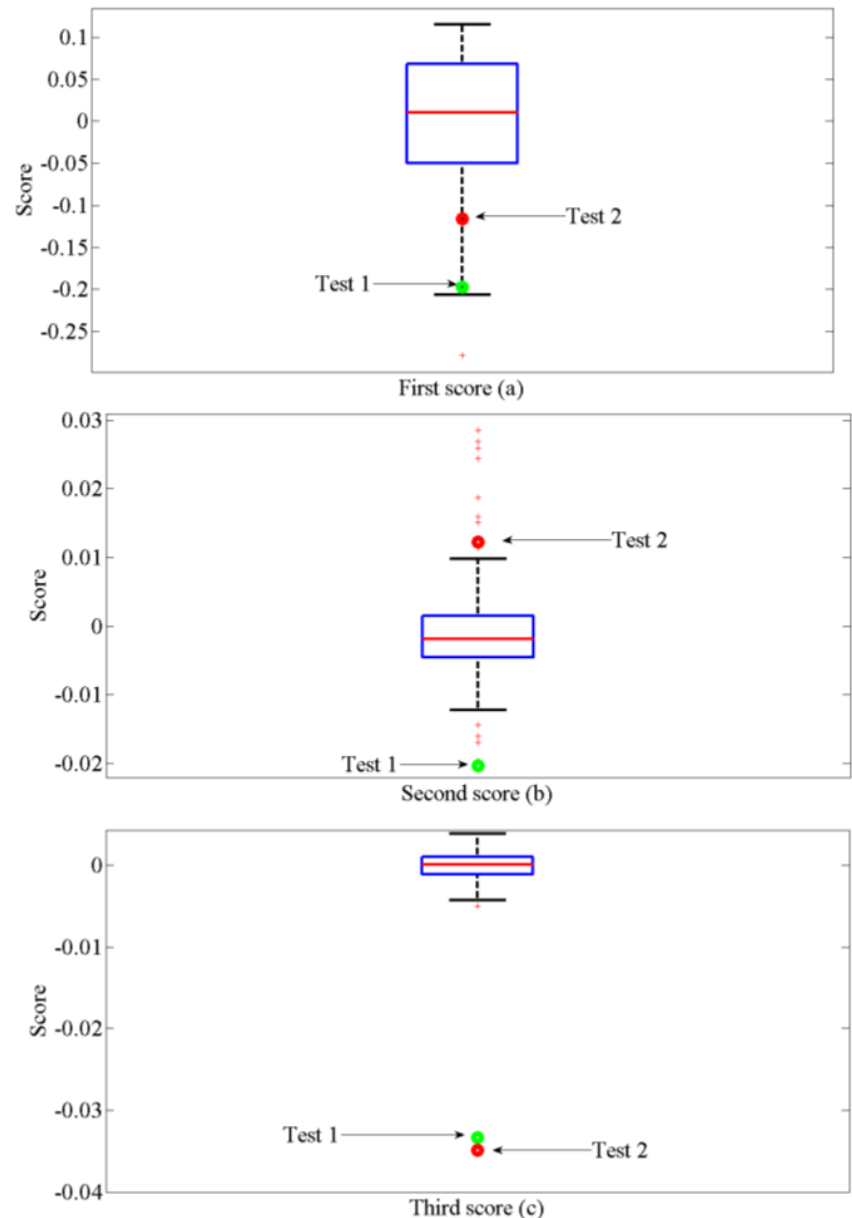

Fig. 16 Box plots of the scores to describe the hoop strain-internal pressure training data generated by using the Ludwik material model and two bi-linear material models for (a) first (b) second and (c) third PLSR scores 


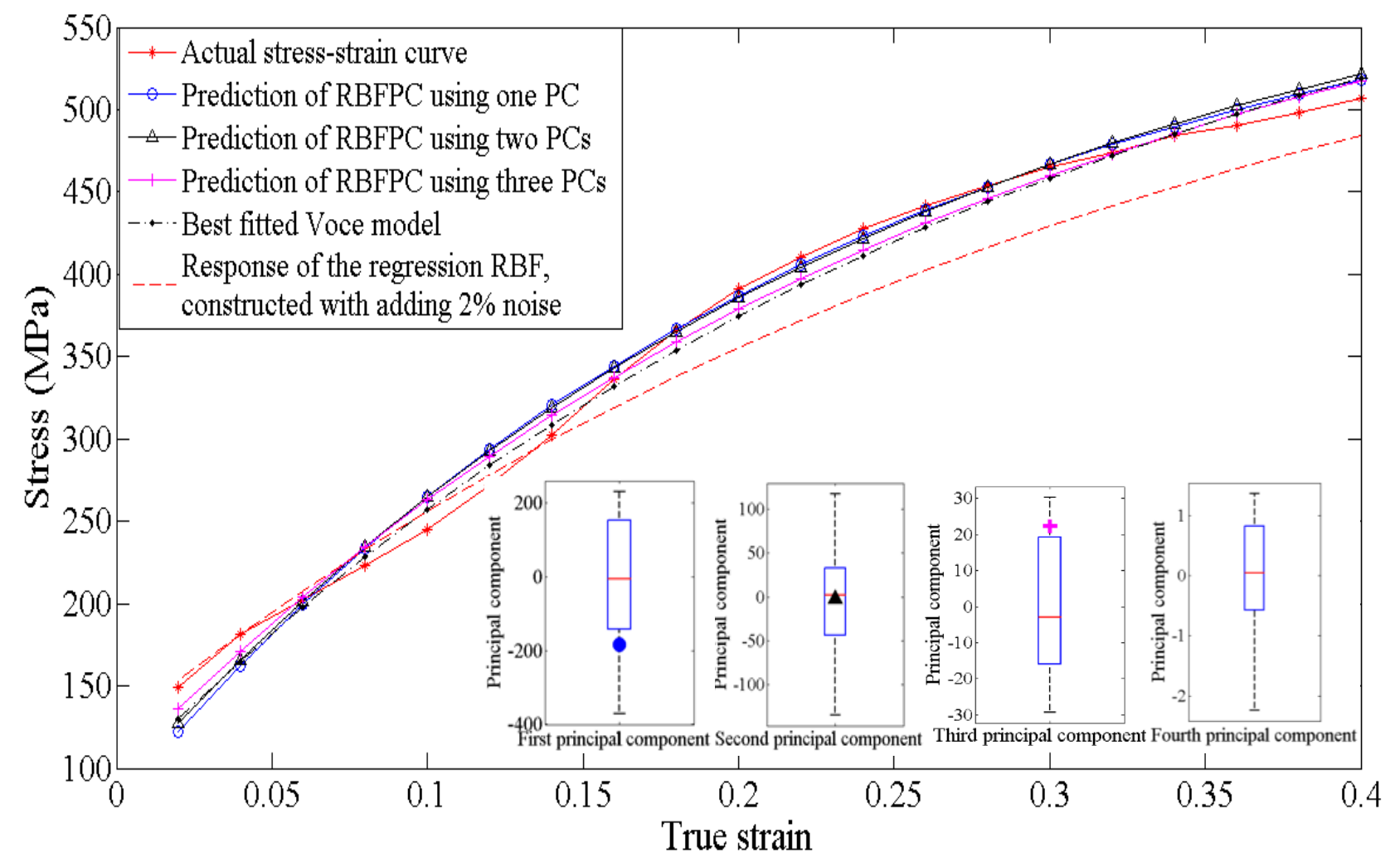

Fig. 17 Stress-strain curves computed from the mapped material parameters using the Voce model in contrast to experimental response and best fitted least squares curve for RBF based approaches

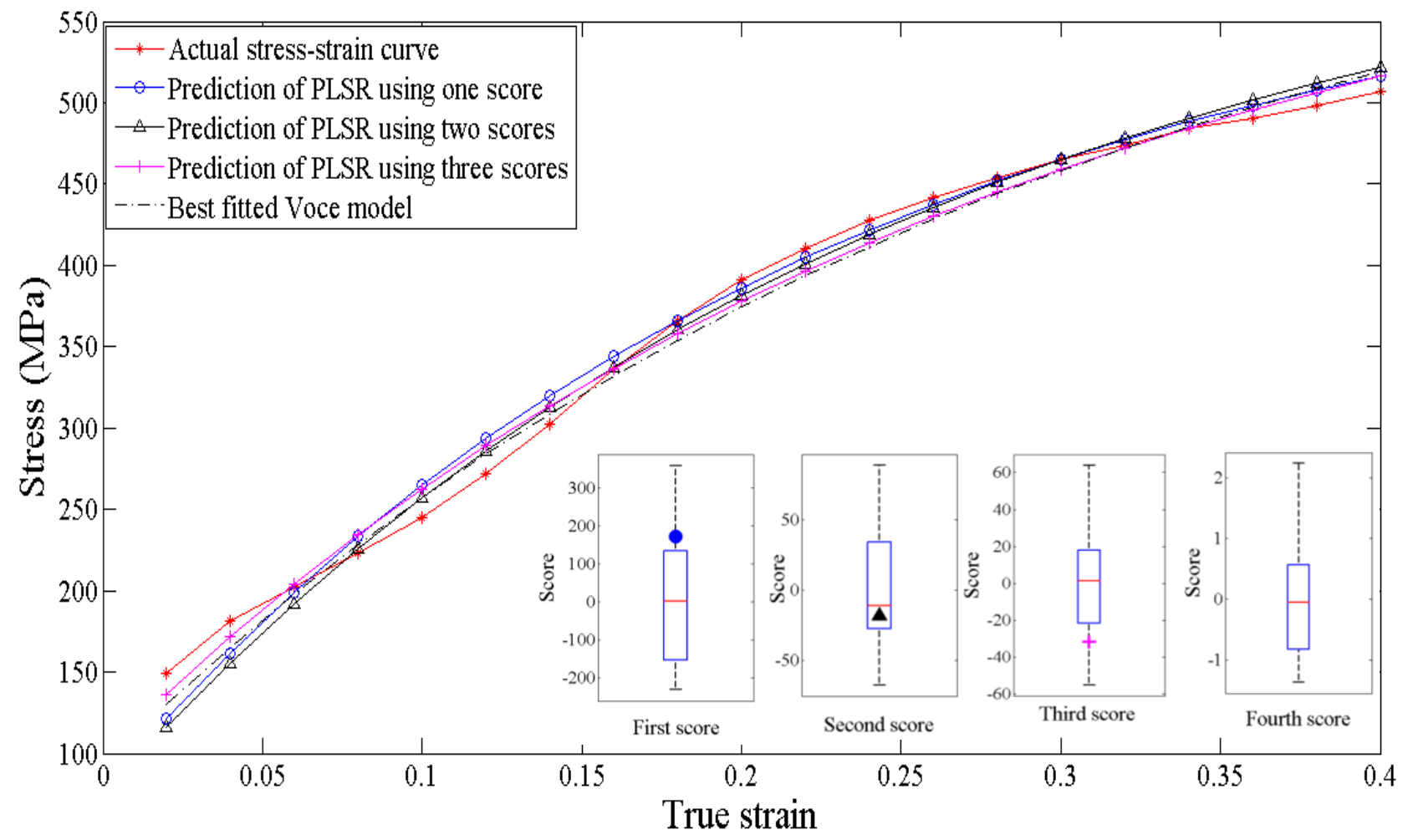

Fig. 18 Stress-strain curves computed from the mapped material parameters using the Voce model in contrast to experimental response and best fitted least squares curve for PLSR based approaches 